\title{
VIRTUAL CUBULATION OF NONPOSITIVELY CURVED GRAPH MANIFOLDS
}

\author{
YI LIU
}

\begin{abstract}
In this paper, we show that an aspherical compact graph manifold is nonpositively curved if and only if its fundamental group virtually embeds into a right-angled Artin group. As a consequence, nonpositively curved graph manifolds have linear fundamental groups.
\end{abstract}

\section{INTRODUCTION}

In this paper, we study virtual properties (i.e. properties that hold for some finite covering) of nonpositively curved graph manifolds. Our main result is the following, (cf. Section 2 for terminology):

Theorem 1.1. Let $M$ be an aspherical compact graph manifold. Then the following statements are equivalent:

(1) $M$ is nonpositively curved;

(2) $M$ is virtually homotopy equivalent to a special cube complex;

(3) $\pi_{1}(M)$ virtually embeds into a finitely generated right-angled Artin group;

(4) $\pi_{1}(M)$ is virtually RFRS.

By the RFRS criterion of Ian Agol for virtual fibering ([Ag1], cf. Subsection 2.5), Theorem 1.1 strengthens a result of Pavel Svetlov that nonpositively curved graph manifolds virtually fibers over the circle, ([Sv], cf. Subsection 2.2). We have also learned recently that Piotr Przytycki and Daniel Wise have an independent proof of the special cubulation for the bounded case using criteria of separability and double-coset separability of surface subgroups, ([PW1]).

It has also been conjectured that 3-manifold groups are linear. Because finitely generated right-angled Artin groups are known to be linear (cf. Subsection 2.4), and because linearity is a commensurability-class property, an interest of Theorem 1.1 is the following:

Corollary 1.2. The fundamental group of any nonpositively curved compact graph manifold is linear.

Theorem 1.1 is motivated by some recent progress in the Virtual Fibering Conjecture (VFC). Back to the 1980s, William Thurston conjectured that every hyperbolic 3-manifold virtually fibers over the circle, ([Th1]). Two years ago, Daniel Wise announced a proof of this conjecture for hyperbolic 3-manifolds that contain essentially embedded geometrically finite closed subsurfaces, using quasiconvex hierarchies ([Wi]). In fact, he showed that every such manifold is virtually

Date: February 8, 2013.

2010 Mathematics Subject Classification. Primary 57M05; Secondary 20 F65. 
homotopy equivalent to a finite-dimensional special cube complex. Hence the fundamental group of any such manifold virtually embeds into a right-angled Artin group. This implies virtual fibering by Agol's criterion. Thus the VFC for hyperbolic 3-manifolds is implied by the Virtual Haken Conjecture (VHC) that every closed 3-manifold virtually contains an (embedded) incompressible subsurface. In more general context, the statement of the VFC is known to be false for many graph manifolds, (cf. Subsection 2.2), and for any closed Seifert-fibered space with nontrivial Euler class of the fiber. Nevertheless, it has been conjectured that the VFC holds for nonpositively curved 3-manifolds, (cf. [Ag1, Conjecture 9.1]). Based on Wise's work and Theorem 1.1, a reasonable specification of this conjecture might be the following ${ }^{1}$ :

Conjecture 1.3. An aspherical compact 3-manifold is nonpositively curved if and only if its fundamental group virtually embeds into a right-angled Artin group.

We explain our strategy to prove Theorem 1.1. It suffices to consider the nontrivial case, namely, where the graph manifold is not itself Seifert fibered. From known results, (cf. Theorems 2.1, 2.6), all the statements of Theorem 1.1 imply virtual fibering. By a trick of doubling the 3-manifold along boundary, and by verifying the Anosov-mapping-torus case directly, we shall focus on the case that $M$ is the mapping torus $M_{\sigma}$ of a multitwist $\sigma$ on an oriented closed surface $F$, (Definition $3.1)$. Our plan is to show (1) $\Rightarrow(2) \Rightarrow(3) \Rightarrow(4) \Rightarrow(1)$, where only the first and the last implications require our effort.

To show virtual RFRS-ness implies nonpositive curving, we introduce the current equations associated to the multitwist $\sigma$. A nondegenerate symmetric solution of this system of equations is virtually ensured by the RFRS condition. Such a solution can be pertubed into a nondegenerate symmetric solution of certain structural equations regarding to nonpositively curved metrics, called the BKN equations. This will provide a nonpositively curved metric on $M_{\sigma}$. To show that nonpositive curving implies a virtual special cubulation, we apply a technique used by Svetlov to find a virtually embedded surface, which virtually guarantees a nondegenerate symmetric solution to the current equations. With such a solution in hand, we shall find a finite collection of first cohomology classes, whose duals are supposably represented by subsurfaces of $M_{\sigma}$ appropriate for the Sageev construction. Keeping this in mind, we shall, however, implement a more explicit construction. It turns out the cubical geometry of the cube complex will be so well understood that one can nicely recognize its virtual specialness.

In Section 2, we provide background materials related to our discussion. In Section 3, we introduce the notion of multitwists and their configuration graphs. We setup the current equations and the BKN equations, and show the virtual equivalence of the existence of their nondegenerate symmetric solutions. In Section 4 , we construct a special cube complex homotopy equivalent to the mapping torus of any multitwist with a bipartite configuration graph. In Section 5, we combine all the partial results above and provide a proof of Theorem 1.1.

\footnotetext{
${ }^{1}$ There has been significant progress on virtual specialization of 3-manifold groups since the announcement of this paper in the fall of 2011. The remaining cases of Conjecture 1.3 are verified by $[\mathrm{Ag} 2, \mathrm{PW} 2]$. The introduction is now a little behind the times but we prefer keeping it as a historical record.
} 
Acknowledgement. The author thanks Ian Agol and Piotr Przytycki for valuable communications, and Stefan Friedl for suggestion on the statement of Theorem 1.1. The author also thanks the referees for proofreading and comments.

\section{Preliminaries}

In this section, we recall some notions and known results relevant to our discussion. As we are only interested in virtual properties in this paper, we shall always work with orientable 3-manifolds for convenience. We refer to [Ja] for standard terminology and facts in 3-manifold topology.

2.1. Nonpositively curved 3-manifolds. A compact 3-manifold is said to be nonpositively curved if it supports a complete Riemannian metric in the interior with everywhere nonpositve sectional curvature. This is equivalent to being virtually nonpositively curved, ([KL]). It is well known that nonpositively curved 3-manifolds are all aspherical.

Atoroidal aspherical 3-manifolds are hyperbolic by the Geometrization, so these are clearly nonpositively curved. A Seifert fibered space is nonpositively curved if and only if it is virtually a product of the circle and a compact surface other than the sphere, ([GW]). A torus bundle with an Anosov monodromy, and hence any Sol-geometric 3-manifold, is not nonpositively curved, ([GW], [Ya]). By a result of Bernhard Leeb, any aspherical compact 3-manifold with either nonempty boundary or at least one atoroidal JSJ piece is nonpositively curved, ([Le]). He also discovered the first family of nontrivial graph manifolds other than the Sol-manifolds which are not nonpositively curved. In [BS], Sergei Buyalo and Pavel Svetlov provided several characterizations of nonpositively curved graph manifolds in terms of certain structure equations and operators associated to the JSJ graph, (cf. Subsection 3.3).

2.2. Graph manifolds. A graph manifold is an irreducible compact orientable 3manifold obtained by gluing up compact Seifert fibered spaces along boundary. We say a graph manifold is nontrivial if it is not a Seifert fibered space, or in other words, if it has a nontrivial JSJ decomposition. Recall that for any orientable irreducible compact 3-manifold, the Jaco-Shalen-Johanson (JSJ) decomposition provides a minimal finite collection of essential tori, canonical up to isotopy, which cuts the manifold into atoroidal and Seifert fibered pieces. Graph manifolds are precisely compact 3-manifolds with only Seifert-fibered pieces. Note that if a 3manifold is virtually a torus bundle with an Anosov monodromy, it is a nontrivial graph manifold according to our convention, but in literature (e.g. [LW]), some authors exclude this case, and use the term 'nontrivial' in a the sense of having nontrivial JSJ decomposition with Seifert-fibered JSJ pieces over hyperbolic bases.

Much has been explored on virtual properties of graph manifolds during the past two decades. Here we are most interested in virtual fibering and nonpositive curving. In [LW], John Luecke and Yingqing Wu exhibited examples of graph manifolds that are not virtually fibered over the circle. There are also graph manifolds that are fibered but not nonpositively curved, for example, the mapping torus $M_{\sigma}$ of an automorphism $\sigma$ of a closed oriented surface $F$ of nonpositive Euler characteristic, where $\sigma$ is the product of positive powers of right-hand Dehn twists along a nonempty collection of disjoint, mutually non-parallel simple closed curves on $F$, ([KL, Theorem 3.7]). However, Pavel Svetlov proved: 
Theorem 2.1 ([Sv], cf. also [WY] for the bounded case). Nonpositively curved graph manifolds virtually fiber over the circle.

Svetlov's proof relies on a criterion of Hyam Rubinstein and Shicheng Wang on the separability of immersed horizontal surfaces in graph manifolds. In [BS], this approach was systematically developed, providing characterizations of various virtual properties of graph manifolds, on three levels in terms of homological, geometric, and operator-spectral data, respectively. For our purpose the second level is particularly interesting, as it describes the nonpositively curved metric on the graph manifold, via the so-called BKN equations. We shall investigate this system of equations in more details in Section 3. The paper [BS] is written in an elegant, expository style, so we recommend it for further information of the reader.

2.3. Special cube complexes. Special cube complexes were introduced by Frédéric Haglund and Daniel Wise (cf. [HW]) as suitable generalizations of graphs in higher dimensions. Heuristically speaking, these are cube complexes in which hyperplanes are 'properly embedded in properly transversal positions' in certain combinatorial sense.

The building blocks in a cube complex are Euclidean $n$-cubes, namely, isometric copies of the double-unit cube $[-1,1]^{n} \subset \mathbf{R}^{n}$ for each dimension $n \geq 0$. For all dimensions $n>0$, every $n$-cube contains exactly $n$ midcubes which are the $(n-1)$ cubes obtained from $[-1,1]^{n}$ intersecting with each coordinate hyperplane through the origin of $\mathbf{R}^{n}$, and $2^{n}$ corners, which are the $n$-simplices each spanned by the half-edges adjacent to a single vertex.

Definition 2.2. A cube complex $X$ is a CW complex whose cells are identified as cubes, such that the attaching maps are combinatorial in the sense that they send the faces of cubes isometrically onto lower-dimensinoal cubes. For any vertex $v \in X$, the $\operatorname{link} \operatorname{lk}(v)$ of $v$ is the complex of simplices over which the union of the corners-of-cubes at $v$ can be naturally regarded as a cone.

A combinatorial map $f: X \rightarrow Y$ between cube complexes is a cellular map, whose restriction to each cube is an isometric homeomorphism onto the image. It is a (combinatorial) local isometry, often written as $f: X \rightarrow Y$, if for any vertex $v \in X$, the induced combinatorial map between links $\dot{f}_{v}: \operatorname{lk}(v) \rightarrow \operatorname{lk}(f(v))$ is furthermore an embedding whose image is a full subcomplex, (i.e. such that a simplex lies in the image whenever all its vertices lie in the image).

Remark. A cube complex is sometimes said to be simple if all the links are simplicial complexes. For square (i.e. 2-dimensional cube) complexes, this is equivalent to saying that no link contains a cycle of at most two edges. Nonpositively curved cube complexes are all simple. A nonpositively curved cube complex is known as a cube complex whose universal covering is $\operatorname{CAT}(0)$ with respect to the naturally induced path metric. An equivalent combinatorial characterization is that all the links are flag (i.e. such that any finite collection of pairwisely joined vertices spans a unique simplex), ([Gr] $)$.

The collection of all the midcubes in a cube complex $X$ forms a new cube complex whose connected components are called hyperplanes. A hyperplane $H$ is two-sided if it naturally induces a local isometry $H \times[-1,1] \rightarrow X$. A hyperplane $H$ is said to be self-intersecting if there are two edges dual to (i.e. across) $H$ lying in a common square of $X$; or it is said to be (directly) self-osculating if there are two dual edges 
not in a common square but adjacent at a common vertex, whose directions towards the vertex induces the same side of $H$ in an obvious sense. Two distinct hyperplanes $H, H^{\prime}$ are said to be inter-osculating if they both intersect and osculate, namely, such that there are two edges dual to $H$ and $H^{\prime}$ respectively, which share a common square of $X$, and that there are two edges dual to $H$ and $H^{\prime}$ respectively, which do not share a common square in $X$ but share a common vertex.

Definition 2.3. A cube complex $X$ is said to be special if its hyperplanes are all two-sided, with no self-intersection, no self-osculation, nor inter-osculation.

Remark. In earlier literatures, (e.g. [HW]), this property was sometimes refered to as being 'A-special', indicating its relation to right-angled $\underline{\text { Artin }}$ groups.

Prototype examples of special cube complexes are graphs and the cube complexes of right-angled Artin groups, (cf. Subsection 2.4). It is also known that every special cube complex can be canonically completed by 'filling up cubes' into a nonpositively curved special cube complex.

2.4. Right-angled Artin groups. Let $\Gamma$ be a (possibly infinite, and possibly locally infinite) simplicial graph. Denote the vertex set as $\operatorname{Ver}(\Gamma)$, and the edge set as $\operatorname{Edg}(\Gamma)$. The right-angled Artin group associated to $\Gamma$ is the group:

$$
A(\Gamma)=\left\langle x_{v}: v \in \operatorname{Ver}(\Gamma) \mid\left[x_{v}, x_{v^{\prime}}\right]:\left\{v, v^{\prime}\right\} \in \operatorname{Edg}(\Gamma)\right\rangle .
$$

Free groups and abelian groups are examples of right-angled Artin groups, associated to edgeless graphs and perfect graphs, respectively.

Right-angled Artin groups are closely related to special cube complexes. For any right-angled Artin group $G$, there is a special nonpositively curved complex $\mathcal{C}(\Gamma)$ by completing the square complex naturally associated to the defining presentation of $A(\Gamma)$. It has a single vertex, and the edges of $\mathcal{C}(\Gamma)$ correspond to the generators $x_{v}$ 's, and the squares of $\mathcal{C}(\Gamma)$ correspond to the relators $\left[x_{v}, x_{v^{\prime}}\right.$ 's. Moreover, Haglund and Wise proved:

Theorem 2.4 ([HW, Theorem 4.2], cf. Remark 2.3). A cube complex is special if and only if it admits a local isometry into the cube complex of a right-angled Artin group.

In fact, the right-angled Artin group associate to a special cube complex $X$ can be canonically constructed by taking the simplicial graph whose vertices are hyperplanes in $X$, such that two vertices are joined by an edge if and only if the corresponding hyperplanes intersect each other. Note Theorem 2.4 does not assume the cube complex to be finite dimensional, nor the right-angled Artin group to be finitely generated.

Finitely generated right-angled Artin groups are known to be subgroups of finitely generated right-angled Coxeter groups, which are subgroups of $\operatorname{SL}(n, \mathbf{Z})$ for some integer $n>0$, (cf. [DJ], [HsW]).

2.5. Agol's RFRS condition. In [Ag1], Ian Agol gave a criterion for 3-manifolds to fiber over the circle in terms of a residual-type condition on the fundamental groups.

Definition 2.5. A group $G$ is said to be residually finite rationally solvable (or $R F R S$ ) if there is a sequence of finite index subgroups $G=G_{0} \triangleright G_{1} \triangleright \cdots$ such that $\cap_{i \geq 0} G_{i}=\{1\}$, and that the quotient homomorphism $G_{i} \rightarrow G_{i} / G_{i+1}$ factors through a free abelian group for every integer $i \geq 0$. 
Examples of virtually RFRS groups are surface groups, reflection groups, and finitely generated right-angled Artin groups, following from the more general fact that finitely generated right-angled Coxeter groups are virtually RFRS ([Ag1, Theorem 2.2]).

Theorem 2.6 (Cf. [Ag1, Theorem 5.1]). Let $M$ be an orientable compact irreducible 3-manifold of zero Euler characteristic. If $\pi_{1}(M)$ is virtually RFRS, then $M$ virtually fibers over the circle.

Roughly speaking, the RFRS assumption allows one to pass to a suitable sequence of finite cyclic coverings by cutting and pasting the 3-manifold along nonseparating subsurfaces. This process can be shown to destroy all the nonproduct parts within a finite number of steps, using Gabai's sutured manifold hierarchy. With his criterion, Agol showed that reflection 3-orbifolds and arithmetic hyperbolic 3-manifolds defined by quadratic forms virtually fiber. His criterion is also an ingredient in Wise's proof of the Virtual Fibering Conjecture for closed Haken hyperbolic 3-manifolds.

\section{Current equations versus BKN equations}

In this section, we introduce the system of the current equations and the system of the BKN equations associated to multitwists. A nondegenerate symmetric solution of the current equations reflects the virtual RFRS-ness of the mapping torus, (Lemma 3.5); and a nondegenerate symmetric solution of the BKN equations reflects the nonpositively-curving property, (Theorem 3.8). We show that the existence of such a solution for any one of these two systems virtually implies that for the other, (Proposition 3.9).

3.1. Mapping tori of multitwists. Let $F$ be an oriented closed surface of negative Euler characteristic. For any essential simple closed curve $z$ on $F$, we denote the (right-hand) Dehn twist on $F$ along $z$ as:

$$
D_{z}: F \rightarrow F .
$$

Note it does not depend on the direction of $z$. It induces the automorphism of $H_{1}(F)$ defined by $D_{z *}(\alpha)=\alpha+I([z], \alpha)[z]$ for any $\alpha \in H_{1}(F)$, where $I: H_{1}(F) \times$ $H_{1}(F) \rightarrow \mathbf{Z}$ denotes the intersection form.

Definition 3.1. Let $F$ be an oriented closed surface of negative Euler characteristic, and $z_{1}, \cdots, z_{s}$ be a collection of mutually disjoint, mutually non-parallel essential simple closed curves, and $b_{1}, \cdots, b_{s}$ be nonzero integers. The multitwist $\sigma$ on $F$ along these curves with multiplicity $b_{i}$ 's is the automorphism (i.e. orientationpreserving self-homeomorphism) defined by $\sigma=D_{z_{1}}^{b_{1}} \cdots D_{z_{s}}^{b_{s}}$. We often more specifically denote a multitwist by the pair $(F, \sigma)$.

The JSJ decomposition of the mapping torus $M_{\sigma}$ of a multitwist $(F, \sigma)$ is fairly easy to describe. Recall that for an oriented closed surface $F$ with an automorphism $\sigma$, the mapping torus $M_{\sigma}$ associated to the pair $(F, \sigma)$ is the closed oriented 3manifold obtained from $F \times[0,1]$ identifying $(x, 0)$ with $(\sigma(x), 1)$ for every $x \in F$. With the notations above, a JSJ torus of $M_{\sigma}$ is the suspension of some $z_{i}$, namely, the image of $z_{i} \times[0,1]$ in $M_{\sigma}$; and a JSJ piece is the suspension of some component of $F-z_{1} \cup \cdots \cup z_{s}$ (i.e. the compact surface obtained from $F$ by removing open regular neighborhoods of $z_{i}^{\prime}$ 's). We shall usually denote the union of JSJ tori of $M_{\sigma}$ 
as $\mathcal{T}$, and the disjoint union of (compact) JSJ pieces as $\mathcal{J}$. There is a naturally induced graph (i.e. a CW 1-complex):

$$
\Lambda=\Lambda(\mathcal{J}, \mathcal{T}),
$$

dual to the decomposition, called the JSJ graph, whose vertex set $\operatorname{Ver}(\Lambda)$ labels to the JSJ pieces, and whose edge set $\operatorname{Edg}(\Lambda)$ labels to the JSJ tori. For any JSJ piece $J_{v} \subset \mathcal{J}$ corresponding to a vertex $v \in \operatorname{Ver}(\Lambda)$, each component of $\partial J$ is a torus $T_{\delta}$ 's labelled by an end-of-edge $\delta$ adjacent to $v$. We often denote the set of ends-of-edges as $\widetilde{\operatorname{Edg}}(\Lambda)$, which may be regarded as a two-fold covering of $\operatorname{Edg}(\Lambda)$. For any end-of-edge $\delta$, there is a natural orientation-reversing homeomorphism:

$$
\phi_{\delta}: T_{\delta} \rightarrow T_{\bar{\delta}}
$$

where $\bar{\delta}$ is the opposite end of the edge which $\delta$ belongs to, satisfying $\phi_{\bar{\delta}}=\phi_{\delta}^{-1}$. These $\phi_{\delta}$ 's together defines an orientation-reversing involution:

$$
\phi: \partial \mathcal{J} \rightarrow \partial \mathcal{J},
$$

often referred to as the gluing. In view of this, we also re-index the curves $z_{i}$ 's and the multiplicity $b_{i}$ 's by $\operatorname{Edg}(\Lambda)$. In the rest of this paper, it will be convenient to write $b_{\delta}$ for $b_{e}$ whenever $\delta$ is an end of $e$. Thus:

$$
b_{\delta}=b_{\bar{\delta}} .
$$

We also index the components of $F-\cup_{e \in \operatorname{Edg}(\Lambda)} z_{e}$ by $\operatorname{Ver}(\Lambda)$, writing $F_{v}$ for the component indexed by $v \in \operatorname{Ver}(\Lambda)$.

We may integrate the defining information for a multitwist alternatively as follows. By a cycle $c$ of $\Lambda$, we mean a sequence of mutually distinct, consecutive directed edges $\vec{e}_{1}, \cdots, \vec{e}_{r}$ such that the terminal endpoint of $\vec{e}_{r}$ coincides with the initial endpoint of $\vec{e}_{1}$. A graph $\Lambda$ is said to be bipartite if every cycle of $\Lambda$ consists of an even number of edges.

Definition 3.2. Let $(F, \sigma)$ be a multitwist. The configuration graph associated to $(F, \sigma)$ is the triple:

$$
\left(\Lambda,\left\{\chi_{v}\right\},\left\{b_{e}\right\}\right),
$$

where $\Lambda$ is the JSJ graph of $M_{\sigma}$, and the integer $\chi_{v}<0$ is the Euler characteristic of $F_{v}$ for each vertex $v \in \operatorname{Ver}(\Lambda)$, and the integer $b_{e} \neq 0$ is the Dehn-twist multiplicity along the curve $z_{e}$ for each edge $e \in \operatorname{Edg}(\Lambda)$. A bipartite configuration graph is such that $\Lambda$ is bipartite.

Remark. It is clear that the positive integer $-\chi_{v}$ is greater than the valence of $v$ minus one. Given a graph decorated with nonzero integers satisfying this compatibility, one may canonically recover a multitwist $(F, \sigma)$. However, it turns out that $\chi_{v}$ 's are irrelevant to the virtual properties of $M_{\phi}$ listed in Theorem 1.1.

Every JSJ piece $J_{v}$ of $M_{\sigma}$ has a natural product structure $F_{v} \times S^{1}$, where $F_{v} \times *$ are parallel to the copy $F_{v} \times\{0\}$, and where $* \times S^{1}$ are the Seifert fibers directed forward along the suspension flow. Thus every (preglue) JSJ torus $T_{\delta} \subset \partial J_{v}$ has a canonical Waldhausen basis, namely:

$$
\left(f_{\delta}, z_{\delta}\right)
$$

where $f_{\delta}$ is the directed Seifert fiber, and $z_{\delta} \subset \partial F_{v}$ is component with the induced orientation. In other words, $\left[f_{\delta}\right]$ and $\left[z_{\delta}\right]$ forms a basis of $H_{1}\left(T_{\delta}\right)$ with the intersection number $I\left(\left[f_{\delta}\right],\left[z_{\delta}\right]\right)=1$. By a straightforward computation, $\phi_{\delta} *: H_{1}\left(T_{\delta}\right) \rightarrow$ 
$H_{1}\left(T_{\bar{\delta}}\right)$ is given by the formula:

$$
\begin{aligned}
& \phi_{\delta *}\left(\left[f_{\delta}\right]\right)=\left[f_{\bar{\delta}}\right]+ \\
& \phi_{\delta *}\left(\left[z_{\delta}\right]\right)= \\
& =
\end{aligned}
$$

In particular, $b_{\delta}$ equals the intersection number between Seifert fibers from the two sides of $T_{\delta}$, namely, $b_{\delta}=I\left(\left[f_{\delta}\right], \phi_{\bar{\delta} *}\left[f_{\bar{\delta}}\right]\right)$ on $H_{1}\left(T_{\delta}\right)$. In fact, the latter is the usual definition of $b_{\delta}\left(\right.$ or $b_{e}$ ) for a graph manifold in general, (cf. [BS, Subsection 1.3]).

For every vertex $v \in \operatorname{Ver}(\Lambda)$, the charge at $v$ is known as the rational number:

$$
k_{v}=\sum_{\delta \in \widehat{\operatorname{Edg}}(v)} \frac{1}{b_{\delta}} .
$$

It is the Euler number of the framing on $\partial J_{v}$ provided by the Seifert fibers from the adjacent pieces, with respect to its own Seifert fibration, or more precisely, the unique $k_{v}$ satisfying $\sum_{\delta \in \widetilde{\operatorname{Edg}}(v)} \frac{\phi_{\bar{\delta} *}\left[f_{\bar{\delta}}\right]}{b_{\delta}}=k_{v} \cdot\left[f_{v}\right]$ in $H_{1}\left(J_{v} ; \mathbf{Q}\right)$, with our notations. The charge at JSJ pieces can be defined for general graph manifolds, but unlike for mapping tori of multitwists, it is not always determined by the intersection numbers $b_{e}$ 's between fibers from both sides on the JSJ tori, (cf. [BS, Subsection 1.3]).

We end up this subsection by pointing out a simplification of our situation. We say a multitwist (or more generally, a surface aumorphism) $(\tilde{F}, \tilde{\sigma})$ covers $(F, \sigma)$ if there is a covering map $\kappa: \tilde{F} \rightarrow F$ such that the diagram below commutes:

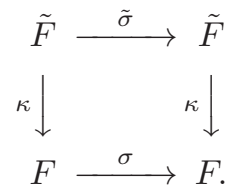

Hence the mapping torus $M_{\tilde{\sigma}}$ covers $M_{\sigma}$ if $(\tilde{F}, \tilde{\sigma})$ covers $(F, \sigma)$.

Lemma 3.3. Every closed, virtually fibered, nontrivial graph manifold is finitely covered by either the mapping torus $M_{\sigma}$ of an Anosov automorphism $\left(T^{2}, \sigma\right)$ of the torus, or the mapping torus $M_{\sigma}$ of a multitwist $(F, \sigma)$ with a bipartite configuration graph.

Proof. If the closed graph manifold is virtually a torus bundle over the circle, it is a nontrivial graph manifold if and only if the monodromy is Anosov. If the virtual fiber has negative Euler characteristic, by the Nielsen-Thurston classification of surface automorphisms ([Th2]), a closed, virtually fibered, nontrivial graph manifold is finitely covered by the mapping torus $M_{\sigma}$ of a nontrivial multitwist $\sigma=D_{z_{1}}^{b_{1}} \cdots D_{z_{s}}^{b_{s}}$ of an oriented closed surface $F$ of negative Euler characteristic. To ensure the bipartition, let $H_{1}(\Lambda) \rightarrow \mathbf{Z}_{2}$ be the homomorphism defined by the parity counting of edges in cycles of $\Lambda$. The kernel of $\pi_{1}(\Lambda) \rightarrow H_{1}(\Lambda) \rightarrow \mathbf{Z}_{2}$ corresponds to a 2 -fold covering $\tilde{\Lambda}$ of $\Lambda$, which furthermore induces a 2-fold covering $\kappa: \tilde{F} \rightarrow F$. Note $\sigma^{\prime}=\sigma^{2}$ acts trivially on $H_{1}\left(F ; \mathbf{Z}_{2}\right)$ as any $D_{z_{i}}^{2}$ does. We may lift each $D_{z_{i}}^{2 b_{i}}$ to a multitwist on $\tilde{F}$ along preimages of $z_{i}$, and hence obtain a lift $\tilde{\sigma}^{\prime}$ of $\sigma^{\prime}$ such that $\tilde{\sigma}^{\prime} \circ \kappa=\kappa \circ \sigma^{\prime}$. Note that $\tilde{\Lambda}$ is bipartite, carrying the configurations of $\left(\tilde{F}, \tilde{\sigma}^{\prime}\right)$, the pair $\left(\tilde{F}, \tilde{\sigma}^{\prime}\right)$ provides a mapping torus as desired.

3.2. The current equations. Let $(F, \sigma)$ be a multitwist with a bipartite configuration graph. In this subsection, we introduce a system of current equations associated to $(F, \sigma)$, whose nondegenerate symmetric solutions give rise to nonseparating subsurfaces of $M_{\sigma}$ intersecting each $z_{e}$ algebraically nontrivially. 
Remember a cycle $c$ of $\Lambda$ is a combinatorial closed path formed by a finite number of consecutive directed edges $\vec{e}_{1}, \cdots, \vec{e}_{r}$. For any cycle $c$, the set of the initial ends of all the $\vec{e}_{i}$ 's will be denoted as $\widehat{\operatorname{Edg}}(c)$.

Definition 3.4. Let $(F, \sigma)$ be a multitwist with a bipartite configuration graph $\left(\Lambda,\left\{\chi_{v}\right\}, b_{v}\right)$. The system of the current equations associated to $(F, \sigma)$, with the unknowns $x_{\delta} \in \mathbf{R}$ where $\delta \in \widetilde{\operatorname{Edg}}(\Lambda)$, consists of the following:

- For any vertex $v$ of $\Lambda$,

$$
\sum_{\delta \in \widehat{\operatorname{dg}}(v)} x_{\delta}=0
$$

- For any cycle $c$ of $\Lambda$,

$$
\sum_{\delta \in \widehat{\operatorname{Edg}}(c)} b_{\delta} x_{\delta}=0
$$

A solution $\left\{x_{\delta}\right\}$ of the current equations is said to be symmetric if $x_{\delta}=-x_{\bar{\delta}}$ for each end-of-edge $\delta$, and to be nondegenerate if no $x_{\delta}$ equals zero.

Remark. As the equations are linear with integral coefficients, there is a nondegenerate symmetric solution over $\mathbf{R}$ if and only if there is such a solution over $\mathbf{Q}$. Somewhat coincidentally, this system of equations is analogous to the Kirchhoff's circuit laws in physics if one allows 'negative resistence', which explains its name.

Lemma 3.5. Let $(F, \sigma)$ be a multitwist with a bipartite configuration graph. Then the current equations associated to $(F, \sigma)$ have a nondegenerate symmetric solution if and only if for each edge $e \in \operatorname{Edg}(\Lambda)$, the simple closed curve $z_{e} \subset F$ is nontrivial in $H_{1}\left(M_{\sigma} ; \mathbf{Q}\right)$ under the natural inclusion of $F$ into the mapping torus $M_{\sigma}$.

Proof. Because $\Lambda$ is bipartite, we may pick a bicoloring $\varepsilon: \operatorname{Ver}(\Lambda) \rightarrow\{ \pm 1\}$, so that vertices of the same color have no edge between each other. This induces directions of the edges, say toward positive vertices, so $z_{e}$ 's are also oriented. As every vertex is adjacent to either only incoming edges or only outgoing edges, a symmetric solution of the current equations at vertices exists if and only if there is a homology class $[s] \in H_{1}(F ; \mathbf{Q})$ with the intersection number $I\left([s],\left[z_{e}\right]\right)=x_{e}$, where $x_{e}$ is the $x_{\delta}$ for the initial end $\delta$ of $e$. When the current equations around cycles are also fulfilled, $\sigma_{*}[s]=[s]+\sum_{e \in \operatorname{Edg}(\Lambda)} b_{e} x_{e}\left[z_{e}\right]$ on $H_{1}(F ; \mathbf{Q})$. This equals $[s]$ because the second term vanishes as it evalutes zero on every cycle. Thus we may suspend $[s] \in H_{1}(F ; \mathbf{Q})$ to be a class $[S] \in H_{2}\left(M_{\sigma} ; \mathbf{Q}\right)$, so that the intersection pairing on $H_{*}\left(M_{\sigma} ; \mathbf{Q}\right)$ gives the intersection number $I\left([S],\left[z_{e}\right]\right)=x_{e}$. Therefore, the existence of a symmetric solution is equivalent to the existence of $[S]$ which yields $x_{e}$ 's under pairing with $\left[z_{e}\right]$ 's, and the solution is nondegenerate if and only if $\left[z_{e}\right.$ ]'s are nontrivial in $H_{1}\left(M_{\sigma} ; \mathbf{Q}\right)$.

An immediate consequence is the following:

Lemma 3.6. Let $(F, \sigma)$ be a multitwist with a bipartite configuration graph. If $M_{\sigma}$ is virtually RFRS, then for some positive integer $m$, and for some multitwist $\left(\tilde{F}, \tilde{\sigma}^{m}\right)$ finitely covering the $m$-th power $\left(F, \sigma^{m}\right)$, the current equations associated to $\left(\tilde{F}, \tilde{\sigma}^{m}\right)$ admit a nondegenerate symmetric solution. 
Proof. By passing to a finite covering, we may assume that the fundamental group of $M=M_{\sigma}$ is itself RFRS. Let $\pi_{1}(M)=G_{0} \triangleright G_{1} \triangleright \cdots$ be a cofinal filtration of finite index subgroups as in Definition 2.5, and let $\tilde{M}_{i}$ be the finite regular covering of $M$ corresponding to $G_{i}$. For any curve $z_{e}$, suppose, as an element of $\pi_{1}(M)$, $z_{e}$ lies in $G_{i}$ but not in $G_{i+1}$, then any component of the preimage of $z_{e}$ in $\tilde{M}_{i}$ is rational homologically nontrivial. Hence this holds for any finite cover of $\tilde{M}_{i}$ as well. As there are finitely many $z_{e}$ 's, we may assume $\tilde{M}_{i}$ is a finite covering such that the components of the preimage of all $z_{e}$ 's are rationally homologically nontrivial. Note that $\tilde{M}_{i}$ is the mapping torus of some automorphism $(\tilde{F}, \tilde{\sigma})$ covering $(F, \sigma)$ of finite degree, where $\tilde{F}$ is the regular covering of $F$ corresponding to the subgroup $\pi_{1}(F) \cap G_{i}$. By further passing to sufficiently high power $\tilde{\sigma}^{m}$ of $\tilde{\sigma}$, we have that $\left(\tilde{F}, \tilde{\sigma}^{m}\right)$ is a multitwist. It has a bipartite configuration graph since this holds for $(F, \sigma)$, so from above we conclude that it has a nondegenerate symmetric solution by Lemma 3.5.

In Figure 1, we have sketched a nondegenerate current on an orientable closed surface with a multitwist. The simple closed curves marked with integers indicate the $z_{e}$ 's and $b_{e}$ 's, while the directed green curves represents a nondegenerate current, by which we mean a first homology class preserved by the multitwist that intersects each $z_{e}$ algebraically nontrivially.

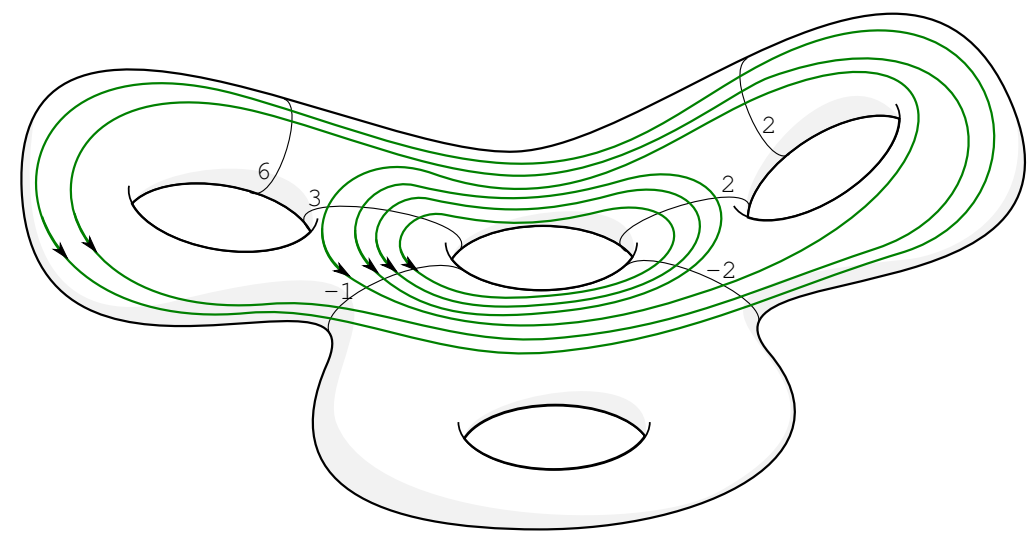

FiguRE 1. A nondegenerate current.

3.3. The BKN equations. For a nontrivial graph manifold, the Buyalo-Kobel'skiuNeumann (BKN) equations are structure equations related to nonpositively curved metrics. Focusing on mapping tori of multitwists, we prefer rearranging them into the following simplified form:

Definition 3.7. Let $(F, \sigma)$ be a multitwist with a bipartite configuration graph $\left(\Lambda,\left\{\chi_{v}\right\},\left\{b_{e}\right\}\right)$. The system of (affine) the BKN equations associated to $(F, \sigma)$, with the unknowns $u_{\delta} \in(0,+\infty)$, and $\gamma_{\delta} \in[-1,1]$, where $\delta \in \widetilde{\operatorname{Edg}}(\Lambda)$, consists of the following:

- For any vertex $v$ of $\Lambda$,

$$
\sum_{\delta \in \widehat{\operatorname{Edg}}(v)} \frac{1-u_{\delta} \gamma_{\delta}}{b_{\delta}}=0 ;
$$


- For any cycle $c$ of $\Lambda$,

$$
\sum_{\delta \in \overparen{\operatorname{Edg}}(c)} \log \left(u_{\delta}\right)=0 .
$$

A solution $\left\{\left(u_{\delta}, \gamma_{\delta}\right)\right\}$ of the BKN equations is said to be symmetric if $u_{\delta}=\frac{1}{u_{\bar{\delta}}}$, and $\gamma_{\delta}=\gamma_{\bar{\delta}}$ for each end-of-edge $\delta$, and to be nondegenerate if no $\gamma_{\delta}$ equals \pm 1 .

Remark. In general, the BKN equations can be defined for any nontrivial graph manifold $M$ with base-orientable JSJ pieces. Let the positive integer $\left|b_{e}\right|$ be the absolute value of the intersection number of fibers from adjacent pieces on $T_{e}$ for each edge $e \in \operatorname{Edg}(\Lambda)$, and the rational number $k_{v}$ be the charge at each vertex $v \in \operatorname{Ver}(\Lambda)$, (cf. Subsection 3.1). With the unknowns $a_{v} \in[0,+\infty)$ for each vertex $v \in \operatorname{Ver}(\Lambda)$, and $\gamma_{e} \in[-1,1]$ for each edge $e \in \operatorname{Edg}(\Lambda)$, the BKN equations are defined as:

$$
k_{v} a_{v}=\sum_{\delta \in \widehat{\operatorname{Edg}}(v)} \frac{a_{v(\bar{\delta})} \gamma_{e(\delta)}}{\left|b_{e(\delta)}\right|},
$$

for every vertex $v \in \operatorname{Ver}(\Lambda)$, where $v(\bar{\delta})$ means the vertex adjacent to the endof-edge $\bar{\delta}$, and $e(\delta)$ means the edge carrying $\delta$, ([BS, Section 3]). We affinize the unknowns by taking $u_{\delta}=\frac{a_{v(\bar{\delta})}}{a_{v(\delta)}}$, and $\gamma_{\delta}=\operatorname{sgn}\left(b_{e(\delta)}\right) \cdot \gamma_{e(\delta)}$ for each end-of-edge $\delta \in \widetilde{\operatorname{Edg}}(\Lambda)$. Note that $M$ virtually fibers if and only if $a_{v}$ 's can be picked to be positive, ([BS, Theorem 3.1]). Note also that $k_{v}=\sum_{\delta \in \widetilde{\operatorname{Edg}}(v)} \frac{1}{b_{\delta}}$ is the formula of the charge for mapping tori of multitwists, (Subsection 3.1). It is clear our definition above is equivalent to the original BKN equations.

Besides several other results, in [BS], Buyalo and Svetlov provided a criterion for a graph manifold to be nonpositively curved. In particular, their result implies:

Theorem 3.8 (Cf. [BS, Theorem 3.1]). Let $(F, \sigma)$ be a multitwist with a bipartite configuration graph. Then the mapping torus $M_{\sigma}$ is nonpositively curved if and only if the BKN equations associated to $(F, \sigma)$ have a nondegenerate symmetric solution. Moreover, in this case, there is a nondegenerate symmetric solution over $\mathbf{Q}$.

To interpretate the unknowns geometrically, every nondegenerate symmetric solution of the affine BKN equations endows $M_{\sigma}$ with a nonpositively curved metric so that the JSJ tori are totally geodesic, Eulidean, with $u_{\delta}$ being the ratio between the lengths of the fibers $f_{\bar{\delta}}$ and $f_{\delta}$, and $\gamma_{\delta}$ being the cosine of the angle between these fibers.

3.4. Nondegenerate symmetric solutions. In this subsection, we study the connection between the current equations and the BKN equations. Heuristically speaking, a multitwist defines a nonpositively curved mapping torus if and only if it virtually admits a nondegenerate current:

Proposition 3.9. Let $(F, \sigma)$ be a multitwist with a bipartite configuration graph. Then the BKN equations associated to $(F, \sigma)$ have a nondegenerate symmetric solution if and only if for some finite covering $(\tilde{F}, \tilde{\sigma})$ of $(F, \sigma)$, the current equations associated to $(\tilde{F}, \tilde{\sigma})$ have a nondegenerate symmetric solution.

We prove Proposition 3.9 in the rest of this subsection. As before, we write the bipartite configuration graph of $(F, \sigma)$ as $\left(\Lambda,\left\{\chi_{v}\right\},\left\{b_{e}\right\}\right)$, and the essential curves on $F$ defining $\sigma$ as $z_{e}$ 's, and the mapping torus of $(F, \sigma)$ as $M_{\sigma}$. 
The 'if' direction follows from perturbing nondegenerate symmetric solutions of the current equations:

Lemma 3.10. If the current equations associated to $(F, \sigma)$ have a nondegenerate symmetric solution, so do the BKN equations.

Proof. Write $k_{v}=\sum_{\delta \in \widetilde{\operatorname{Edg}}(\Lambda)} \frac{1}{b_{\delta}}$ for the charge at a vertex $v \in \operatorname{Ver}(\Lambda)$. If all the charges $k_{v}$ 's are zero, there is clearly a nondegenerate symmetric solution $\left\{\left(u_{\delta}^{*}, \gamma_{\delta}^{*}\right)\right\}$ to the BKN equations, where $u_{\delta}^{*}=1$ and $\gamma_{\delta}^{*}=0$. In the following, we suppose that not all the charges are zero.

Let $\left\{x_{\delta}^{*}\right\}$ be a nondegenerate symmetric solution of the current equations. With the new parameters $t_{v}$ 's and $\omega_{\delta}$ 's in $\mathbf{R}$, we put:

$$
u_{\delta}=\exp \left(t_{v(\delta)}-t_{v(\bar{\delta})}\right) \text {, and } \gamma_{\delta}=\cos \left(\sqrt{\omega_{\delta}+\omega_{\bar{\delta}}}\right) \text {, }
$$

where $v(\delta)$ denotes the vertex that $\delta$ is adjacent to, and where $\cos (\sqrt{z})$ stands for the analytic function $\sum_{j=0}^{\infty} \frac{(-1)^{j}}{(2 j) !} z^{j}$. Then the BKN equations around cycles are automatically satisfied.

To ensure the BKN equations at vertices, consider the functions:

$$
W_{v}(\vec{t}, \vec{\omega})=\sum_{\delta \in \widehat{\operatorname{Edg}}(v)} \frac{1-\exp \left(t_{v}-t_{v(\bar{\delta})}\right) \cos \left(\sqrt{\omega_{\delta}+\omega_{\bar{\delta}}}\right)}{b_{\delta}},
$$

for each vertex $v \in \operatorname{Ver}(\Lambda)$, where $\vec{t}$ and $\vec{\omega}$ are the vectors with the coordinates $t_{v}$ 's and $\omega_{\delta}$ 's, respectively. Formally, these together define a smooth map:

$$
W: \mathbf{R}^{\operatorname{Ver}(\Lambda)} \times \mathbf{R}^{\widetilde{\operatorname{Edg}}(\Lambda)} \rightarrow \mathbf{R}^{\operatorname{Ver}(\Lambda)},
$$

sending the origin to the origin. Our goal is to use the existence of $\left\{x_{\delta}^{*}\right\}$ to show that for some $\vec{t}$ sufficiently close to the origin, there are $\vec{\omega}$ with sufficiently small positive coordinates, fulfilling $W_{v}(\vec{t}, \vec{\omega})=0$ for any vertex $v \in \operatorname{Ver}(\Lambda)$.

First observe that $W$ is a submersion near the origin. Indeed:

$$
\left.\mathrm{d} W_{v}\right|_{O}=\sum_{\delta \in \overline{\operatorname{Edg}}(v)} \frac{-2 \mathrm{~d} t_{v}+2 \mathrm{~d} t_{v(\bar{\delta})}+\mathrm{d} \omega_{\delta}+\mathrm{d} \omega_{\bar{\delta}}}{2 b_{\delta}},
$$

so the tangent map:

$$
\left.\mathrm{d} W\right|_{O}: T_{O}\left(\mathbf{R}^{\operatorname{Ver}(\Lambda)} \times \mathbf{R}^{\widetilde{\operatorname{Edg}}(\Lambda)}\right) \rightarrow T_{W(O)} \mathbf{R}^{\operatorname{Ver}(\Lambda)},
$$

satisfies at the origin:

$$
\begin{aligned}
\mathrm{d} W\left(\frac{\partial}{\partial t_{v}}\right) & =-k_{v} \frac{\partial}{\partial y_{v}}+\sum_{\delta \in \widehat{\operatorname{Edg}}(v)} \frac{1}{b_{\delta}} \frac{\partial}{\partial y_{v(\bar{\delta})}} \\
\mathrm{d} W\left(\frac{\partial}{\partial \omega_{\delta}}\right) & =\frac{1}{2 b_{\delta}} \frac{\partial}{\partial y_{v(\delta)}}+\frac{1}{2 b_{\delta}} \frac{\partial}{\partial y_{v(\bar{\delta})}}
\end{aligned}
$$

Here the $y_{v}$ 's stand for the natural coordinate parameters of the target space $\mathbf{R}^{\operatorname{Ver}(\Lambda)}$. To see $\left.\mathrm{d} W\right|_{O}$ is surjective, note that by our assumption, there is some vertex $u \in \operatorname{Ver}(\Lambda)$ at which the charge $k_{u}$ nonzero. Since the image of $\frac{\partial}{\partial t_{u}}-$ $2 \sum_{\delta \in \widetilde{\operatorname{Edg}}(u)} \frac{\partial}{\partial \omega_{\delta}}$ under $\left.\mathrm{d} W\right|_{O}$ equals $-2 k_{u} \frac{\partial}{\partial y_{u}}$, we see $\frac{\partial}{\partial y_{u}} \in \operatorname{Im}\left(\left.\mathrm{d} W\right|_{O}\right)$. Thus for any vertex $v$ adjacent to $u, \frac{\partial}{\partial y_{v}}$ also lies in $\operatorname{Im}\left(\left.\mathrm{d} W\right|_{O}\right)$ by the formula of $\mathrm{d} W\left(\frac{\partial}{\partial \omega_{\delta}}\right)$ for $\delta \in \widetilde{\operatorname{Edg}}(v)$. Further applying the formula of $\mathrm{d} W\left(\frac{\partial}{\partial \omega_{\delta}}\right)$ 's for other end-of-edges, 
it follows from the connectivity of the graph $\Lambda$ that for all $v \in \operatorname{Ver}(\Lambda)$, $\frac{\partial}{\partial y_{v}}$ lies in $\operatorname{Im}\left(\left.\mathrm{d} W\right|_{O}\right)$. This means $\left.\mathrm{d} W\right|_{O}$ is surjective. Now as $W$ is a submersion near the origin, the preimage of the origin of $\mathbf{R}^{\operatorname{Ver}(\Lambda)}$ is a smooth submanifold $\mathcal{V}$ of $\mathbf{R}^{\operatorname{Ver}(\Lambda)} \times \mathbf{R}^{\widetilde{\operatorname{Edg}(\Lambda)}}$ through the origin, of the dimension $|\widetilde{\operatorname{Edg}}(\Lambda)|$.

Because $\left\{x_{\delta}^{*}\right\}$ satisfies the current equations around cycles, it is clear that there exist $l_{v}^{*} \in \mathbf{R}$, for all $v \in \operatorname{Ver}(\Lambda)$, such that $b_{\delta} x_{\delta}^{*}$ equals $l_{v(\delta)}^{*}-l_{v_{(\delta)}}^{*}$ for each endof-edge $\delta \in \widetilde{\operatorname{Edg}}(\Lambda)$. Consider the curve $\alpha:(-\epsilon, \epsilon) \rightarrow \mathbf{R}^{\operatorname{Ver}(\Lambda)} \times \mathbf{R}^{\widetilde{\operatorname{Edg}(\Lambda)}}$, where $\alpha(s)=(\vec{t}(s), \vec{\omega}(s))$ is defined by:

$$
t_{v}(s)=l_{v}^{*} s, \text { and } \omega_{\delta}(s)=\frac{1}{2} \arccos ^{2}\left(\frac{1}{\cosh \left(b_{\delta} x_{\delta}^{*} s\right)}\right) .
$$

Since $\left\{x_{\delta}^{*}\right\}$ is symmetric, i.e. $x_{\bar{\delta}}^{*}=-x_{\delta}^{*}$, we have:

$$
\begin{aligned}
W_{v}(\alpha(s)) & =\sum_{\delta \in \widehat{\operatorname{Edg}}(v)} \frac{1-\exp \left(b_{\delta} x_{\delta}^{*} s\right) \cdot\left(\cosh \left(b_{\delta} x_{\delta}^{*} s\right)\right)^{-1}}{b_{\delta}} \\
& =-\sum_{\delta \in \overparen{\operatorname{Edg}}(v)} \frac{\tanh \left(b_{\delta} x_{\delta}^{*} s\right)}{b_{\delta}} .
\end{aligned}
$$

Using the current equation at vertices, it is clear that all the $W_{v}(\alpha(s))$ vanish up to the second order, namely, $W_{v}(\alpha(s))=o\left(s^{2}\right)$. Thus there exists a curve $\alpha^{*}:(-\epsilon, \epsilon) \rightarrow \mathcal{V}$ which is jet-2 equivalent to $\alpha$. Hence $\alpha^{*}(s)=\left(\vec{t}^{*}(s), \vec{\omega}^{*}(s)\right)$ has the Taylor expansion near zero:

$$
t_{v}^{*}(s)=l_{v}^{*} s+o\left(s^{2}\right), \text { and } \omega_{\delta}^{*}(s)=\frac{\left(b_{\delta} x_{\delta}^{*}\right)^{2} s^{2}}{2}+o\left(s^{2}\right) .
$$

Because $\left\{x_{\delta}^{*}\right\}$ is nondegenerate, $\omega_{\delta}^{*}(s)$ is strictly positive for any sufficiently small nonzero $s$. Therefore, for any sufficiently small nonzero $s$, the corresponding $u_{\delta}^{*}=\exp \left(t_{v(\delta)}^{*}(s)-t_{v(\bar{\delta})}^{*}(s)\right)$ and $\gamma_{\delta}^{*}=\cos \left(\sqrt{\omega_{\delta}^{*}(s)+\omega_{\bar{\delta}}^{*}(s)}\right)$ gives a nondegenerate symmetric solution of the BKN equations.

To prove the 'only-if' direction, we need some ingredient from [Sv]:

Lemma 3.11. If the BKN equations associated to $(F, \sigma)$ has a nondegenerate symmetric solution over $\mathbf{Q}$, then there are linearly independent elements $\left[\theta_{\delta}^{+}\right],\left[\theta_{\delta}^{-}\right] \in$ $H_{1}\left(T_{\delta} ; \mathbf{Q}\right)$ for each end-of-edge $\delta \in \widetilde{\operatorname{Edg}}(\Lambda)$, subject to $\left[\theta_{\delta}^{ \pm}\right]= \pm \phi_{\bar{\delta} *}\left(\left[\theta_{\bar{\delta}}^{ \pm}\right]\right)$, and there is a connected immersed orientable closed surface $j: S \rightarrow M_{\sigma}$ satisfying the following. For the preimage $j^{-1}\left(T_{\delta}\right)$ of each $T_{\delta}$, the homology class $j_{*}\left[j^{-1}\left(T_{\delta}\right)\right]$ is a scalar multiple of $\left[\theta_{\delta}\right]=\left[\theta_{\delta}^{+}\right]+\left[\theta_{\delta}^{-}\right]$, and for each component $\hat{\theta} \subset j^{-1}\left(T_{\delta}\right)$, the homology class $j_{*}[\hat{\theta}]$ is a scalar multiple of either $\left[\theta_{\delta}^{+}\right]$or $\left[\theta_{\delta}^{-}\right]$. Moreover, $S$ is a virtual fiber, namely, there is a surface bundle finitely covering $M_{\sigma}$ with the fiber covering $S$ up to homotopy.

Proof. We outline the proof in our context following [Sv], (cf. Remark 3.3 for the translation of the notations). Suppose $\left\{\left(u_{\delta}, \gamma_{\delta}\right)\right\}$ is a nondegenerate symmetric solution over $\mathbf{Q}$ of the BKN equations associated to $(F, \sigma)$. In fact, the elements $\left[\theta_{\delta}^{ \pm}\right]$can be explicitly picked as:

$$
\left[\theta_{\delta}^{ \pm}\right]=\frac{1 \pm \gamma_{\delta}}{2 b_{\delta}}\left(\phi_{\bar{\delta} *}\left[f_{\bar{\delta}}\right] \pm u_{\delta}\left[f_{\delta}\right]\right) .
$$


Note these are linearly independent elements as $u_{\delta}>0$ and $-1<\gamma_{\delta}<1$. Moreover, it is straightforward to check the following, where $\left[\theta_{\delta}\right]=\left[\theta_{\delta}^{+}\right]+\left[\theta_{\delta}^{-}\right]$, and $I(\cdot, \cdot)$ denotes the intersection pairing on $H_{1}\left(T_{\delta} ; \mathbf{Q}\right)$ :

- For each end-of-edge $\delta \in \widetilde{\operatorname{Edg}}(\Lambda)$, using the nondegeneracy:

$$
I\left(\left[f_{\delta}\right],\left[\theta_{\delta}^{ \pm}\right]\right)>0
$$

- For each end-of-edge $\delta \in \widetilde{\operatorname{Edg}}(\Lambda)$ :

$$
I\left(\left[f_{\delta}\right],\left[\theta_{\delta}\right]\right)=1
$$

- For each vertex $v \in \operatorname{Ver}(\Lambda)$, using the BKN equation at $v$ and the formula of the charge $k_{v}$, (cf. Subsection 3.1):

$$
\sum_{\delta \in \widehat{\operatorname{Edg}}(v)} I\left(\left[\theta_{\delta}\right], \frac{\phi_{\bar{\delta} *}\left[f_{\bar{\delta}}\right]}{b_{\delta}}\right)=k_{v} ;
$$

- And for each end-of-edge $\delta \in \widetilde{\operatorname{Edg}}(\Lambda)$ :

$$
\left|\frac{I\left(\phi_{\bar{\delta} *}\left[f_{\bar{\delta}}\right],\left[\theta_{\delta}^{ \pm}\right]\right)}{I\left(\left[f_{\delta}\right],\left[\theta_{\delta}^{ \pm}\right]\right)}\right|=u_{\delta}
$$

The first three properties imply the existence of a connected, horizontal (i.e. transverse to the fiber in each Seifert fibered piece), immersed, orientable closed surface $j: S \leftrightarrow M_{\sigma}$. Furthermore, one may require that for any component $\hat{\theta} \subset j^{-1}\left(T_{\delta}\right)$, the homology class $j_{*}[\hat{\theta}]$ is a scalar multiple of either $\left[\theta_{\delta}^{+}\right]$or $\left[\theta_{\delta}^{-}\right]$, and $j_{*}\left[j^{-1}\left(T_{\delta}\right)\right]$ is a scalar multiple of $\left[\theta_{\delta}\right]$, ([Ne, Lemma 3.1]; cf. [Sv, Proposition 1.4]). The last property together with the BKN equations around cycles imply that $S$ is a virtually embedded horizontal surface, and hence is a virtual fiber, using a cocycle criterion due to Rubinstein and Wang ([RW]; cf. [Sv, Proposition 1.4]).

Lemma 3.12. If the BKN equations associated to $(F, \sigma)$ have a nondegenerate symmetric solution, so do the current equations associated to some finite covering $(\tilde{F}, \tilde{\sigma})$ of $(F, \sigma)$.

Proof. By Theorem 3.8, we may assume the nondegenerate symmetric solution to be over Q. Let $j: S \rightarrow M_{\sigma}$ be the virtual fiber surface as ensured by Lemma 3.11. Note that for any essential simple closed curve $z_{\delta} \subset T_{\delta}$ corresponding to an end-ofedge $\delta \in \widetilde{\operatorname{Edg}}(\Lambda)$, at least one of the intersection numbers $I\left(\left[z_{\delta}\right],\left[\theta_{\delta}^{ \pm}\right]\right)$is nonzero. Suppose $\kappa: \tilde{M}_{\sigma} \rightarrow M_{\sigma}$ is a regular finite covering in which a finite covering $\tilde{S}$ of $S$ embeds as a fiber. Then every component $\tilde{z}$ of $\kappa^{-1}\left(z_{e}\right)$ intersects a component of the preimage $\kappa^{-1}(j(S)$ ), i.e. a conjuate of $\tilde{S}$ under the covering transformation, algebraically nontrivially. In particular, $\tilde{z}$ is nontrivial in $\tilde{M}_{\sigma}$. Note that any component $\tilde{F}$ of $\kappa^{-1}(F)$ is still a fiber of $\tilde{M}_{\sigma}$. Then a further finite covering of $\tilde{M}_{\sigma}$ is a mapping torus associated to a multitwist $(\tilde{F}, \tilde{\sigma})$. Now the defining essential curves of $\tilde{\sigma}$ cover the $z_{e}$ 's, so they are nontrivial in $H_{1}\left(M_{\tilde{\sigma}} ; \mathbf{Q}\right)$. By Lemma 3.5, this is to say the current equations associated to $(\tilde{F}, \tilde{\sigma})$ have a nondegenerate symmetric solution.

Proof of Proposition 3.9. We apply Lemma 3.10 to $(\tilde{F}, \tilde{\sigma})$ in the 'if' direction. Note the mapping torus $M_{\tilde{\sigma}}$ is nonpositively curved if and only if so is $M_{\sigma}$, ([KL]), thus equivalently, the BKN equations associated to $(\tilde{F}, \tilde{\sigma})$ have a nondegenerate 
symmetric solution if and only if so do the BKN equations associated to $(F, \sigma)$, (Theorem 3.8). The 'only-if' direction follows from Lemma 3.12.

\section{Virtual SPECial CUbUlation}

In this section, we construct a virtual special cube complex homotopy equivalent to the mapping torus of a multitwist with a bipartite configuration graph, under the assumption that the associated current equation possesses a nondegenerate symmetric solution, (Proposition 4.1). As mentioned before, this assumption should be regarded as a seemingly weaker translation of the RFRS condition.

Proposition 4.1. Let $(F, \sigma)$ be a multitwist with a bipartite configuration graph. Suppose the current equations associated to $(F, \sigma)$ have a nondegenerate symmetric solution. Then for some integer $0 \leq n \leq|\operatorname{Ver}(\Lambda)|-2$, the product $M_{\sigma} \times \mathbf{R}^{n}$ of the mapping torus $M_{\sigma}$ and the $n$-space $\mathbf{R}^{n}$ is homeomorphic to a virtually special cube complex.

Remark. In fact, the special cube complex by our construction is complete with finitely many hyperplanes, but when $n>0$, it has no compact convex subcomplex homotopy equivalent to $\tilde{M}_{\sigma}$.

We prove Proposition 4.1 in the rest of this section. Our cubulation is inspired by the Sageev construction. Generally speaking, from any finite collection of $\pi_{1}$ injective surfaces, the Sageev construction allows one to obtain a CAT(0) cube complex on which the fundamental group of the 3-manifold acts, (cf. [Ni]). This will be a proper action if one can find a 'sufficient' collection, and the virtual specialness will be verified if one can show the separability and the double-coset separability of the surface subgroups. This approach has been taken by Piotr Przytycki and Dani Wise recently, ([PW1]). Instead of that, in this paper, we shall present an explicit construction, through which the virtual specialness can be seen directly.

Throughout this section, we suppose $(F, \sigma)$ satisfies the assumption of Proposition 4.1. We shall write the configuration graph of as $\left(\Lambda,\left\{\chi_{v}\right\},\left\{b_{e}\right\}\right)$, and the essential curves on $F$ defining $\sigma$ as $z_{e}$ 's. Regarding the mapping torus $M_{\sigma}$ as $F \times[0,1]$ identifying $(x, 0)$ with $(\sigma(x), 1)$ for every $x \in F$. there is a natural inclusion:

$$
F \subset M_{\sigma},
$$

identifying $F$ as the copy $F \times\{0\}$ in $M_{\sigma}$. The Poincaré dual of $[F] \in H_{2}\left(M_{\sigma}\right)$ will be written as:

$$
\alpha_{F} \in H^{1}\left(M_{\sigma}\right) .
$$

4.1. The cut-bind system $(\mathcal{Z}, \mathcal{W})$. In this subsection, we choose some setup data for the construction of the cubulation. It will be described as a cut-bind system $(\mathcal{Z}, \mathcal{W})$ of curves with respect to $(F, \sigma)$, which naturally induces a cohomology class $\bar{\xi} \in H^{1}(F)$ and a finite collection of lifted cohomology classes $\xi^{1}, \cdots, \xi^{n+2} \in$ $H^{1}\left(M_{\sigma}\right)$ for some $0 \leq n \leq|\operatorname{Ver}(\Lambda)|-2$.

Definition 4.2. Suppose $\mathcal{Z} \subset F$ is a collection of mutually disjoint simple closed curves so that $\mathcal{P}=F-\mathcal{Z}$ is a disjoint union of (compact) pairs-of-pants. We say the pants decomposition $\mathcal{P}=F-\mathcal{Z}$ is subordinate to $(F, \sigma)$ if $z_{e}$ is a component of $\mathcal{Z}$ for every $e \in \operatorname{Edg}(\Lambda)$. The components of $\mathcal{Z}$ are called the cut-curves. 
Definition 4.3. Suppose $\mathcal{P}=F-\mathcal{Z}$ is a pants decomposition of $F$ subordinate to $(F, \sigma)$, and $\mathcal{W} \subset F$ is a union of oriented mutually-disjoint simple closed curves, representing a cohomology class $\bar{\xi} \in H^{1}(F)$ in Poincaré dual. If for every cut curve $z \in \mathcal{Z}, \mathcal{W} \cap z$ is nonempty consisting of exactly $|\bar{\xi}([z])|$ points, and if for every pairof-pants $P \subset \mathcal{P}, \mathcal{W} \cap P$ is a union of disjoint arcs, then we call $(\mathcal{Z}, \mathcal{W})$ a cut-bind system with respect to $(F, \sigma)$. The components of $\mathcal{W}$ are called the bind-curves.

Lemma 4.4. With the notations above, there is a pants decomposition $\mathcal{P}=F-\mathcal{Z}$ subordinate to $(F, \sigma)$, so that every cut-curve $z \subset \mathcal{Z}$ represents a nontrivial element (up to sign) in $H_{1}\left(M_{\sigma} ; \mathbf{Q}\right)$. In particular, every cut-curve $z \subset \mathcal{Z}$ is non-separating on $F$, adjacent to two distinct pairs-of-pants of $\mathcal{P}$.

Proof. Let $\mathcal{Z}_{0}$ be the union of the $z_{e}$ 's. By Lemma 3.5, as the current equations associated to $(F, \sigma)$ have a nondegenerate solution, each $z_{e}$ survives in $H_{1}\left(M_{\sigma}\right)$. Suppose by induction that for some $k \geq 0, \mathcal{Z}_{k} \subset F$ is a union of mutually nonparallel, disjoint essential simple closed curves that has been constructed, so that every component $z \subset \mathcal{Z}$ survives in $H_{1}\left(M_{\sigma} ; \mathbf{Q}\right)$.

Whenever $F-\mathcal{Z}_{k}$ is not a disjoint union of pairs-of-pants, we construct $\mathcal{Z}_{k+1}$ as follows. If there is a non-planar component of $C$ of $F-\mathcal{Z}_{k}$, let $z \subset C$ be a nonseparating simple closed curve. It is clear that $z$ is rational-homologically nontrivial in $M_{\sigma}$, so we take $\mathcal{Z}_{k+1}=\mathcal{Z}_{k} \cup\{z\}$. Otherwise, there is still a component planar $C$ of $F-\mathcal{Z}_{k}$ which has at least four boundary components. With the orientation induced from that of $C$, at least two boundary components $z^{\prime}, z^{\prime \prime} \subset \partial C$ such that $\left[z^{\prime}\right]+\left[z^{\prime \prime}\right] \neq 0$ in $H_{1}\left(M_{\sigma} ; \mathbf{Q}\right)$. Let $z \subset C$ be a simple closed curve separating $z^{\prime} \cup z^{\prime \prime}$ from the other components of $\partial C$, so $z$ is rational-homologically nontrivial in $M_{\sigma}$. We take $\mathcal{Z}_{k+1}=\mathcal{Z}_{k} \cup\{z\}$.

This process terminates after finitely many steps. In the end, we get a pants decomposition $\mathcal{P}=F-\mathcal{Z}$ as desired.

Fix a pants decomposition $\mathcal{P}=F-\mathcal{Z}$ subordinate to $(F, \sigma)$ as asserted by Lemma 4.4.

Lemma 4.5. With the notations above, there is a cohomology class $\bar{\xi} \in H^{1}(F)$ invariant under $\sigma_{*}$, such that $\bar{\xi}([z])$ is nontrivial for any cut-curve $z \subset \mathcal{Z}$.

Proof. As the pants decomposition $\mathcal{P}=F-\mathcal{Z}$ satisfies the conclusion of Lemma 4.4, we may find a generic $\xi^{0} \in H^{1}\left(M_{\sigma} ; \mathbf{Q}\right)$, so that $\xi^{0}([z]) \neq 0$ for any cutting curve $z \subset \mathcal{Z}$. After passing to a multiple, we may assume $\xi^{0} \in H^{1}\left(M_{\sigma}\right)$ as well. Take $\bar{\xi} \in H^{1}(F)$ to be the restriction of $\xi^{0}$ under the natural inclusion $F \subset M_{\sigma}$. It is straightforward to check that $\bar{\xi}$ satisfies the conclusion.

Fix a cohomology class $\bar{\xi} \in H^{1}(F)$ as asserted by Lemma 4.5.

Lemma 4.6. With the notations above, there is a cut-bind system $(\mathcal{Z}, \mathcal{W})$ with respect to $(F, \sigma)$ such that $[\mathcal{W}] \in H_{1}(F)$ is in Poincaré dual to $\bar{\xi} \in H^{1}(F)$.

Proof. Let $P \subset \mathcal{P}$ be a pair-of-pants. For simplicity, we write $v$ for the vertex $v(P) \in \operatorname{Ver}(\Lambda)$ carrying a pair-of-pants $P \subset \mathcal{P}$, and write $i$ for the index $i(v) \in$ $\{1,2, \cdots, n+2\}$. Note that the value of the cohomology class $\bar{\xi}$ on the three components $z, z^{\prime}, z^{\prime \prime}$ of $\partial P$ (with the induced orientations) are three nonzero integers $m, m^{\prime}, m^{\prime \prime}$, respectively, so that $m+m^{\prime}+m^{\prime \prime}=0$. Without loss of generality, we assume $m^{\prime}$ and $m^{\prime \prime}$ has the same sign, and $m$ has the opposite sign. Thus one may take $\left|m^{\prime}\right|$ parallel arcs from $z$ to $z^{\prime}$, and $\left|m^{\prime \prime}\right|$ parallel arcs from $z$ to $z^{\prime \prime}$, so that they 
are mutually disjoint, cutting $P$ into one octagon and $|m|-2$ bands. See Figure 2 for an illustration with $m=5, m^{\prime}=-2$, and $m^{\prime \prime}=-3$, in which the bind-arcs are colored green.

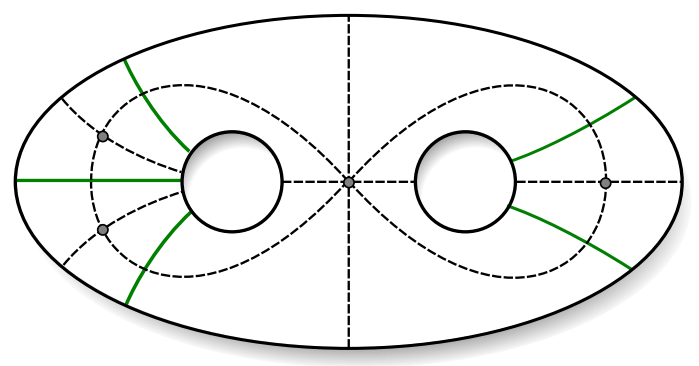

FiguRE 2. Bind-arcs in a pair-of-pants and the dual cubulation.

There is a canonical orientation for these arcs so that their union is dual to the restriction $\left.\bar{\xi}\right|_{P} \in H^{1}(P)$. These parallel arcs will later serve as the bind-arcs $\mathcal{W} \cap P$. Moreover, we glue up these $P$ 's according to the pants decomposition, or more precisely, embed $P$ into $F$, so that the bind-arcs match up along the boundary, giving rise to bind-curves $\mathcal{W} \subset F$. Arbitrary matching up may result in some $[\mathcal{W}] \in H_{1}(F)$ which differs from the dual of $\bar{\xi} \in H^{1}(F)$ by an integral linear combination of the $[z]^{\prime}$ 's, where $z \subset \mathcal{Z}$ are the cut-curves. However, we may modify the matching by shifting the embedding of $P$ 's into $F$ near $\partial P$, so that $[\mathcal{W}]$ is the Poincaré dual of $\bar{\xi}$, as desired.

Fix a cut-bind system $(\mathcal{Z}, \mathcal{W})$ with respect to $(F, \sigma)$ as asserted by Lemma 4.6.

Lemma 4.7. With the notations above, there are canonical integral cohomology classes:

$$
\xi^{1}, \cdots, \xi^{n+2} \in H^{1}\left(M_{\sigma}\right)
$$

for some $0 \leq n \leq|\operatorname{Ver}(\Lambda)|-2$, such that each $\xi^{j}$ restricts to $\bar{\xi} \in H^{1}(F)$ under the natural inclusion $F \subset M_{\sigma}$, and that for every vertex $v \in \operatorname{Ver}(\Lambda)$, there is exactly one $\xi^{i}$ with $\xi^{i}\left(\left[f_{v}\right]\right)=0$.

Proof. As $\bar{\xi} \in H^{1}(F)$ is invariant under $\sigma_{*}$, it comes from the restriction of some $\xi^{0} \in H^{1}\left(M_{\sigma}\right)$, and the space of such cohomology classes is exactly $\xi^{0}+\mathbf{Z} \alpha_{F}$. For any vertex $v \in \operatorname{Ver}(\Lambda)$, and any $\xi(t)=\xi^{0}+t \alpha_{F}$, we have $\xi\left(\left[f_{v}\right]\right)=0$ if and only if $t$ equals $-\xi^{0}\left(\left[f_{v}\right]\right)$, since $\alpha_{F}\left(\left[f_{v}\right]\right)=1$. We may take $\xi^{1}, \cdots, \xi^{n+2}$ to be all the $\xi(t)$ 's, where $t=-\xi^{0}\left(\left[f_{v}\right]\right)$ as $v$ runs over $\operatorname{Ver}(\Lambda)$. This is the canonically (ordered) finite set of cohomology classes.

Let $\xi^{1}, \cdots, \xi^{n+2} \in H^{1}\left(M_{\sigma}\right)$ be the cohomology as asserted by Lemma 4.7. It will be convenient to write:

$$
i(v) \in\{1,2, \cdots, n+2\},
$$

for the unique index associated to $v \in \operatorname{Ver}(\Lambda)$. We often call $i(v)$ the vertical index with respect to $v$, and call any other index $j$ a horizontal index with respect to $v$. 
4.2. The cube complex $X$. In this subsection, we construct a canonical cube complex $X$ provided a choice of the cut-bind $\operatorname{system}(\mathcal{Z}, \mathcal{W})$ with respect to $(F, \sigma)$, as can be obtained by Subsection 4.1.

Let $\bar{\xi} \in H^{1}(F)$ and $\xi^{1}, \cdots, \xi^{n+1} \in H^{1}\left(M_{\sigma}\right)$ be the canonically associated cohomology classes as before. We also have a natural cubulation of $F$ dual to $(\mathcal{Z}, \mathcal{W})$, namely, the cubulation obtained by matching up the dual cubulation on each pairof-pants. See Figure 2 for an illustration, where the dots and the dashed arcs are the vertices and the edges in the dual cubulation, respectively. Note that the boundary of the pair-of-pants is regarded as a union of mid-cubes.

Step 1. Cubulate $J_{v} \times \mathbf{R}^{n}$. For every vertex $v \in \operatorname{Ver}(\Lambda)$, the JSJ piece $J_{v}$ is the mapping torus of $\sigma$ restricted to $F_{v} \subset F$. It has a canonical Seifert fibered structure given by the short exact sequence of groups:

$$
1 \longrightarrow \pi_{1}\left(f_{v}\right) \longrightarrow \pi_{1}\left(J_{v}\right) \stackrel{p_{v}}{\longrightarrow} \pi_{1}\left(F_{v}\right) \longrightarrow 1,
$$

and a natural inclusion $F_{v} \subset J_{v}$. Hence $\pi_{1}\left(J_{v}\right)$ is a canonical direct product $\pi_{1}\left(F_{v}\right) \times \pi_{1}\left(f_{v}\right)$.

Let $\widehat{F}_{v}$ be the universal covering of $F_{v}$ with the induced cubulation. We parametrize $\mathbf{R}^{n+2}$ by the real coordinates $\vec{\lambda}=\left(\lambda^{1}, \cdots, \lambda^{n+2}\right)$, and for each $1 \leq i \leq n+2$, let $\mathbf{R}_{(i)}^{n+1}$ be the subspace defined by $\lambda^{i}=0$. We often denote points of $\mathbf{R}_{(i)}^{n+1}$ by $\vec{\lambda}_{(i)}=\left(\lambda^{1}, \cdots, \widehat{\lambda^{i}}, \cdots, \lambda^{n+2}\right)$. The integral lattice of $\mathbf{R}^{n+2}$ endows $\mathbf{R}^{n+2}$, and each $\mathbf{R}_{(i)}^{n+1}$, with a standard cubulation given by the tiling of standard unit $(n+2)$-cubes. Therefore, the fundamental group $\pi_{1}\left(J_{v}\right)$ acts on:

$$
\widehat{X}_{v}=\widehat{F}_{v} \times \mathbf{R}_{(i(v))}^{n+1},
$$

via:

$$
g \cdot\left(w, \vec{\lambda}_{(i(v))}\right)=\left(p_{v}(g) \cdot w, \vec{\lambda}_{(i(v))}+\vec{\xi}_{(i(v))}([g])\right),
$$

for any $g \in \pi_{1}\left(J_{v}\right)$, and any $\left(w, \vec{\lambda}_{(i(v))}\right) \in \widehat{X}_{v}$. Here $p_{v}(g)$ acts by the deck transformations on $\widehat{F}_{v}$, and $\vec{\xi}_{(i(v))}([g])$ denotes $\left.\left.\left(\xi^{1}([g]), \cdots, \overline{\xi^{i(v)}([g]}\right]\right), \cdots, \xi^{n+2}([g])\right)$, where $[g] \in H_{1}\left(J_{v}\right)$ is the corresponding homology class.

Lemma 4.8. The action on $\pi_{1}\left(J_{v}\right)$ on the cube complex $\widehat{X}_{v}$ is proper and combinatorial. The quotient cube complex:

$$
X_{v}=\pi_{1}\left(J_{v}\right) \backslash \widehat{X}_{v},
$$

is homeomorphic to $J_{v} \times \mathbf{R}^{n}$. Moreover, there is a canonical isomorphism $\pi_{1}\left(X_{v}\right) \cong$ $\pi_{1}\left(J_{v}\right)$ induced by an n-plane bundle structure of $X_{v}$ over $J_{v}$.

Proof. The stabilizer of $\pi_{1}\left(J_{v}\right)$ at any point of $\widehat{F}_{v}$ is the center $\pi_{1}\left(f_{v}\right) \cong \mathbf{Z}$. Since $f_{v}$ acts on $\mathbf{R}_{(i(v))}^{n+1}$ as a translation by $\vec{\xi}_{(i(v))}\left(\left[f_{v}\right]\right)$, which is nontrivial by Lemma 4.7, the action of $\pi_{1}\left(J_{v}\right)$ is proper on $\widehat{F} \times \mathbf{R}_{(i(v))}^{n+1}$. It clearly preserves the cube complex structure as well.

Moreover, let $V_{v}$ be the subspace of $\mathbf{R}_{(i(v))}^{n+1}$ spanned by $\vec{\xi}_{(i(v))}\left(\left[f_{v}\right]\right)$ over $\mathbf{R}$. Then the $n$-dimensional foliation of $\mathbf{R}_{(i(v))}^{n+1}$ by the affine parallel copies of any subspace complementary $U$ to $V_{v}$ induces an (affine) $n$-plane bundle structure of $X_{v}$ over $J_{v}$. In fact, different choices of $U$ 's result in naturally isomorphic bundles over $J_{v}$. This bundle is trivial because the structure group is translational, and it induces a canonical isomorphism $\pi_{1}\left(X_{v}\right) \cong \pi_{1}\left(J_{v}\right)$. 
For any end-of-edge $\delta \in \widetilde{\operatorname{Edg}}(\Lambda)$ adjacent to $v$, we write:

$$
\partial_{\delta} X_{v} \subset \partial X_{v}
$$

for the boundary component corresponding to $\delta$. It is a cube complex formed by mid-cubes, and $\pi_{1}\left(\partial_{\delta} X_{v}\right) \cong \pi_{1}\left(T_{\delta}\right)$ under the natural isomorphism $\pi_{1}\left(X_{v}\right) \cong$ $\pi_{1}\left(J_{v}\right)$. To understand the cubulation on $\partial_{\delta} X_{v}$, remember we have cubulated $z_{\delta}$ as the quotient of $\mathbf{R}^{1}$ by a translation by $\xi^{i(v)}\left(\left[z_{\delta}\right]\right)$. Thus, the universal covering of $\partial_{\delta} X_{v}$ can be naturally regarded as $\mathbf{R}^{n+2}$ up to integral translations, where $\mathbf{R}^{n+2} \cong \mathbf{R}^{1} \times \mathbf{R}_{(i(v))}^{n+1}$ is identified via plugging $\mathbf{R}^{1}$ as the $i(v)$-th factor. Using this identification, $\partial_{\delta} X_{v}$ is the quotient of $\mathbf{R}^{n+2}$ under the action of $\pi_{1}\left(T_{e}\right)$ via:

$$
g \cdot \vec{\lambda}=\vec{\lambda}+\vec{\xi}([g]),
$$

for any $g \in \pi_{1}\left(T_{\delta}\right)$, and any $\vec{\lambda} \in \mathbf{R}^{n+2}$. Here $\vec{\xi}([g])$ denotes $\left(\xi^{1}([g]), \cdots, \xi^{n+2}([g])\right)$. In particular, any $z_{\delta}$ translates by $\vec{\Delta}\left(\bar{\xi}\left(\left[z_{\delta}\right]\right)\right)=\left(\bar{\xi}\left(\left[z_{\delta}\right]\right), \cdots, \bar{\xi}\left(\left[z_{\delta}\right]\right)\right)$ along the diagonal of $\mathbf{R}^{n+2}$, and any $f_{v}$ translates only in the subspace $\mathbf{R}_{(i(v))}^{n+1}$.

Because integral translations on the $\mathbf{R}_{(i(v))}^{n+1}$ factor of $\widehat{X}_{v}=\widehat{F}_{v} \times \mathbf{R}_{(i(v))}^{n+1}$ commute with the action of $\pi_{1}\left(J_{v}\right)$ on $\widehat{X}_{v}$, this induces a group of automorphisms of the cube complex $X_{v}$. It is an abelian group naturally isomorphic to the quotient of $\mathbf{Z}^{n+1}$ modulo $\vec{\xi}_{(i(v))}\left(\left[f_{v}\right]\right)$. We shall call these automorphisms combinatorial translations of $X_{v}$.

Step 2. Cubulate $M_{\sigma} \times \mathbf{R}^{n}$. It is almost clear how to glue up these $X_{v}$ 's. In the previous step, we identified $\partial_{\delta} X_{v}$ up to combinatorial translations as the quotient of $\mathbf{R}^{n+2}$ by the action of $\pi_{1}\left(T_{\delta}\right)$, and under the identification, the action of $\pi_{1}\left(T_{\delta}\right)$ and $\pi_{1}\left(T_{\bar{\delta}}\right)$ coincide on $\mathbf{R}^{n+2}$. This implies the cubulations on the boundary components corresponding to any pair of opposite ends match up. Therefore, we may glue up all $X_{v}$ 's along the boundaries with respect to $\Lambda$, matching up the cube complex structures, to obtain a cube complex:

$$
X=\bigcup_{v \in \operatorname{Ver}(\Lambda)} X_{v}
$$

which is clearly complete.

However, the cube complex $X$ is determined only up to combinatorial translations along the boundary at this point. To further make the matching canonical, we need to specify a reference point on each component of each $\partial X_{v}$. For this purpose, let:

$$
\bar{h}: \Lambda \rightarrow F,
$$

be a lift of the configuration graph $\Lambda$ into $F$, namely, such that $\bar{r} \circ \bar{h}: \Lambda \rightarrow \Lambda$ is homotopic to the identity where $\bar{r}: F \rightarrow \Lambda$ is the natural collapsing. This gives a base-point $\bar{\nu}_{v}=\bar{h}(v)$ on each $F_{v}$, and a base-point $\bar{\nu}_{e}=\bar{h}(e) \cap z_{e}$ on each $z_{e}$, and a path $\bar{\gamma}_{\delta}=\bar{h}(e(\delta)) \cap F_{v(\delta)}$ from $\bar{\nu}_{v(\delta)}$ to $\bar{\nu}_{e(\delta)}$ for each end-of-edge $\delta \in \widetilde{\operatorname{Edg}}(\Lambda)$, where $v(\delta) \in \operatorname{Ver}(\Lambda)$ is the vertex adjacent to $\delta$, and $e(\delta) \in \operatorname{Edg}(\Lambda)$ is the edge carrying $\delta$. We may also assume that the $\bar{\nu}_{v}$ 's and $\bar{\nu}_{e}$ 's are vertices of the cubulated $F_{v}$, and hence they miss the bind-curves $\mathcal{W}$. By picking a direction of all the edges of $\Lambda$, it makes sense to speak of the algebraic intersection number of any $\bar{\gamma}_{\delta}$ with the bind-curves $\mathcal{W}$, which will be ad hoc written as $\bar{\xi}\left(\bar{\gamma}_{\delta}\right)$. For every vertex $v \in \operatorname{Ver}(\Lambda)$, let:

$$
\tilde{\nu}_{v}=\left(\widehat{\nu}_{v}, \overrightarrow{0}\right)
$$


be the point in $\widehat{X}_{v}=\widehat{F}_{v} \times \mathbf{R}_{(i(v))}^{n+1}$, where $\widehat{\nu}_{v} \in \widehat{F}_{v}$ is a lift of $\bar{\nu}_{v} \in F_{v}$. Thus the lift of $\bar{h}(\Lambda) \cap F_{v}$ into $\widehat{F}_{v}$ based at $\widehat{\nu}_{v}$ gives rise to the $\widehat{\nu}_{\delta} \in \widehat{F}_{v}$ for every end-of-edge $\delta \in \widetilde{\operatorname{Edg}}(v)$ adjacent to $v$. Let:

$$
\tilde{\nu}_{\delta}=\left(\widehat{\nu}_{\delta}, \vec{\Delta}_{(i(v))}\left(\bar{\xi}\left(\bar{\gamma}_{\delta}\right)\right)\right),
$$

be the further lift $\bar{\nu}_{e(\delta)}$ into $\widehat{X}_{v}$, where $\vec{\Delta}_{(i(v))}: \mathbf{R} \rightarrow \mathbf{R}_{(i(v))}^{n+1}$ is the diagonal inclusion, namely, $\vec{\Delta}_{(i(v))}(t)=(t, \cdots, t)$ for any $t \in \mathbf{R}$. This naturally gives rise to a lift of $\bar{h}(\Lambda) \cap F_{v}$ into $\widehat{F}_{v} \times \mathbf{R}_{(i(v))}^{n+1}$ based at $\tilde{\nu}_{v}$, for instance, using the linear extension on the $\mathbf{R}_{i(v)}^{n+1}$ factor. We denote the corresponding images in the quotient as:

$$
\nu_{v} \in X_{v}, \text { and } \nu_{\delta} \in \partial_{\delta} X_{v(\delta)},
$$

which are all vertices in the cubulation.

Glue up the $X_{v}$ 's matching the reference points on the boundary. We denote the gluing by:

$$
\Phi_{\delta}: \partial_{\delta} X_{v(\delta)} \rightarrow \partial_{\bar{\delta}} X_{v(\bar{\delta})},
$$

for every end-of-edge $\delta \in \widetilde{\operatorname{Edg}}(\Lambda)$, satisfying $\Phi_{\bar{\delta}}=\Phi_{\delta}^{-1}$, and $\Phi_{\delta}\left(\nu_{\delta}\right)=\nu_{\bar{\delta}}$. Thus we obtain a cube complex $X$, and in addition, a lifted $h: \Lambda \rightarrow X$ such that $r \circ h: \Lambda \rightarrow \Lambda$ is the identity, where $r: X \rightarrow \Lambda$ is the natural collapsing.

Lemma 4.9. Different choices of $\bar{h}: \Lambda \rightarrow F$ and the $\widehat{\nu}_{v}$ 's in $\widehat{F}$ yield the same cube complex $X$ up to combinatorial isometry.

Proof. Suppose we choose a new lift of the configuration graph $\bar{h}^{\prime}: \Lambda \rightarrow F$ with a new collection of lifted base-points $\widehat{\nu}_{v}^{\prime} \in \widehat{F_{v}}$. Denote the gluing map with respect to the new reference points by $\Phi_{\delta}^{\prime}: \partial_{\delta} X_{v(\delta)} \rightarrow \partial_{\bar{\delta}} X_{v(\bar{\delta})}$. We need to find a combinatorial isometry $\tau_{v}: X_{v} \rightarrow X_{v}$ for each $v \in \operatorname{Ver}(\Lambda)$, such that the following commutative diagram holds for every end-of-edge $\delta \in \widetilde{\operatorname{Edg}}(\Lambda)$ :

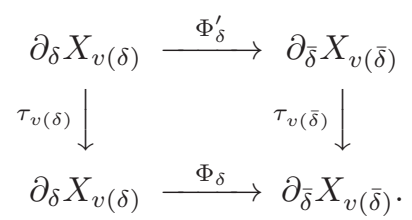

To obtain the $\tau_{v}$ 's, let each $\widehat{\beta}_{v}$ be an immersed path in $\widehat{F}_{v}$ from $\widehat{\nu}_{v}$ to $\widehat{\nu}_{v}^{\prime}$, and let $\bar{\beta}_{v}$ be the projected immersed path in $F_{v}$. We define:

$$
\tau_{v}: X_{v} \rightarrow X_{v}
$$

to be the combinatorial translation induced from the integral translation on $\widehat{X}_{v}$ given by:

$$
\tilde{\tau}_{v} \cdot\left(w, \vec{\lambda}_{(i(v))}\right)=\left(w, \vec{\lambda}_{(i(v))}+\vec{\Delta}_{(i(v))}\left(\bar{\xi}_{\left.\left(\bar{\beta}_{v}\right)\right)}\right) .\right.
$$

It is straightforward to check that these $\tau_{v}$ 's satisfy the diagram above. To be precise, let each $\bar{\beta}_{e}$ be an immersed path in $z_{e}$ from $\bar{\nu}_{e}$ to $\bar{\nu}_{e}^{\prime}$, and let $\widehat{\beta}_{\delta}$ be the lifted path in $\widehat{F}_{v}$ based at $\widehat{\nu}_{\delta}$, where $\delta$ is the end-of-edge adjacent to $v$ and carried by $e$. We further lift $\widehat{\beta}_{\delta}$ as a path $\tilde{\beta}_{\delta}$ in $\widehat{X}_{v}$ based at $\tilde{\nu}_{v}$, so that the $\mathbf{R}_{(i(v))}^{n+1}$ component of the other end-point $\tilde{\nu}_{\delta}^{*}$ is $\vec{\Delta}_{(i(v))}\left(\bar{\xi}\left(\bar{\beta}_{e}\right)\right)$. Let $g \in \pi_{1}\left(F_{v}\right)$ be the element represented by the loop obtained from joining the consecutive directed paths $-\bar{\beta}_{e},-\bar{\gamma}_{\delta}, \bar{\beta}_{v}$ and 
$\bar{\gamma}_{\delta}^{\prime}$. We regard $g$ as in $\pi_{1}\left(J_{v}\right)$ via the natural inclusion induced by $F_{v} \subset J_{v}$. Then from the construction, $\vec{\xi}_{(i(v))}([g])=\vec{\Delta}(\bar{\xi}([g]))$. Moreover, we have:

$$
\tilde{\tau}_{v} \cdot \tilde{\nu}_{\delta}^{\prime}=g \cdot \tilde{\nu}_{\delta}^{*} .
$$

This means in the quotient space $X_{v}, \tau_{v(\delta)} \cdot \nu_{\delta}^{\prime}=\nu_{\delta}^{*}$. However, from the construction we also have $\Phi_{\delta}\left(\nu_{\delta}^{*}\right)=\nu_{\bar{\delta}}^{*}$. Hence it is verified that $\Phi_{\delta} \circ \tau_{v(\delta)}=\tau_{v(\delta)} \circ \Phi_{\delta}^{\prime}$.

Hence the cube complex $X$ is canonically determined by the multitwist $(F, \sigma)$ and the cut-bind system $(\mathcal{Z}, \mathcal{W})$.

Lemma 4.10. The cube complex $X$ is homeomorphic to $M_{\sigma} \times \mathbf{R}^{n}$ with a canonical isomorphism $\pi_{1}(X) \cong \pi_{1}\left(M_{\sigma}\right)$.

Proof. Under the natural inclusion $F \subset M_{\sigma}$, the reference points also gives rise to natural reference points for the JSJ pieces and JSJ tori of $M_{\sigma}$. Thus the caonical isomorphisms $\pi_{1}\left(X_{v}\right) \cong \pi_{1}\left(M_{v}\right)$ induces a canonical isomorphism $\pi_{1}(X) \cong \pi_{1}\left(M_{\sigma}\right)$ using the graph-of-groups decomposition. To see $X$ is homeomorphic to $M_{\sigma} \times \mathbf{R}^{n}$, we take an $n$-plane foliation of $X_{v}$ for each $v \in \operatorname{Ver}(\Lambda)$ as in the proof of Lemma 4.8. For any $e \in \operatorname{Edg}(\Lambda)$, the foliations on the quotient of $\mathbf{R}^{n+2}$ modulo $\pi_{1}\left(T_{e}\right)$ induced from the two adjacent pieces are clearly isotopic in the space of (affine) $n$-plane foliations. $X$ is topologically a trivial $n$-plane bundle over $M_{\sigma}$. Hence $X$ is homeomorphic to $M_{\sigma} \times \mathbf{R}^{n}$.

4.3. Hyperplanes in $X$. In this subsection, we study the cubical geometry of the hyperplanes of $X$. This will prepare us for the proof of the virtual specialness of $X$.

There are $n+3$ types hyperplanes in $X$, one called the cut-type and the others called the bind-types, indexed by $j \in\{1,2, \cdots, n+2\}$. With the notations from Subsection 4.2, an edge of the cubulation of $F$ is said to have the cut-type (resp. the bind-type) if it is dual to a cut-curve (resp. a bind-curve). This also gives rise to types of edges in any covering space of $F$ with the induced cubulation. In the subcomplex $X_{v}$ corresponding to a vertex $v \in \operatorname{Ver}(\Lambda)$, a cut-type edge is an edge projected from a cut-type edge parallel to $\widehat{F}$ in $\widehat{F} \times \mathbf{R}_{(i(v))}^{n+1}$; and a bind-type edge of index $j$, is either an edge projected from a bind-type edge parallel to $\widehat{F}$ in $\widehat{F} \times \mathbf{R}_{(i(v))}^{n+1}$, if $j$ is the vertical index $i(v)$, or an edge projected from an edge parallel to $\mathbf{R}_{(i(v))}^{n+1}$, if $j$ is a horizontal index. By our construction, it is clear that every hyperplane is dual to exactly one type of edges. This gives rise to $n+3$ types of hyperplanes.

Lemma 4.11. The cut-type hyperplanes in $X$ are in natural bijection to the cutcurves on $F$, namely, the components of $\mathcal{Z}$. Moreover, for every cut-type hyperplane is two-sided, homeomorphic to $T^{2} \times \mathbf{R}^{n}$. Here $T^{2}$ denotes the torus.

Proof. Let $z \subset \mathcal{Z}$ be a cut-curve, and $v \in \operatorname{Ver}(\Lambda)$ be one of the at most two vertices for which $z \subset F_{v}$ (possibly on the boundary). Then for any copy of the universal covering $\widehat{z}$ of $z$ in $\widehat{F}_{v}$, the stabilizer of $\widehat{z} \times \mathbf{R}_{(i(v))}^{n+1}$ is clearly a conjugate of $\pi_{1}\left(T_{z}\right)$ in $\pi_{1}\left(J_{v}\right)$, where $T_{z} \subset M_{\sigma}$ denotes the mapping torus $M_{\sigma \mid z}$ of $\sigma$ restricted to $z$, which is a torus. Thus the quotient hyperplane $H_{z} \subset X$ is two-sided, homeomorphic to $T^{2} \times \mathbf{R}^{n}$. Moreover, different choices of the copies projects to the same $H_{z}$, and every cut-type hyperplane $H \subset X$ is naturally some $H_{z}$ by the definition. This 
gives the natural bijection between cut-type hyperplanes of $X$ and the cut-curves on $F$.

We denote the union of all the cut-type hyperplanes in $X$ as $\mathcal{H}_{\mathcal{Z}}$, and denote the component of $\mathcal{H}_{\mathcal{Z}}$ corresponding to the cut-curve $z \subset \mathcal{Z}$ as $H_{z}$. It is also clear that the components of $X-\mathcal{H}_{\mathcal{Z}}$ are in natural bijection to the pairs-of-pants on $F$, namely, the components of $\mathcal{P}=F-\mathcal{Z}$; moreover, each component is homeomorphic to the product of a pair-of-pants with $S^{1} \times \mathbf{R}^{n}$. We denote:

$$
X_{\mathcal{P}}=X-\mathcal{H}_{\mathcal{Z}}
$$

and denote the component corresponding to the pair-of-pants $P \subset \mathcal{P}$ as $X_{P}$. This induces a graph-of-spaces decomposition of $X$, whose dual graph:

$$
\Upsilon=\Upsilon(\mathcal{P}, \mathcal{Z})
$$

is naturally isomorphic to the graph dual to the pants decomposition $\mathcal{P}=F-\mathcal{Z}$ of $F$. This decomposition also naturally agrees with the decomposition of $M_{\sigma}$ :

$$
\mathcal{J}_{\mathcal{P}}=M_{\sigma}-\mathcal{T}_{\mathcal{Z}}
$$

where $\mathcal{T}_{\mathcal{Z}}$ is the disjoint union of the mapping tori $T_{z}=M_{\sigma \mid z}$ of $\sigma$ restricted to each cut-curve $z \subset \mathcal{Z}$, and $\mathcal{J}_{\mathcal{P}}$ is the disjoint union of of the mapping tori $J_{P}=M_{\sigma \mid P}$ of $\sigma$ restricted to each pair-of-pants $P \subset \mathcal{P}$. As the pants decomposition is subordinate to $(F, \sigma), \Upsilon$ refines the configuration graph $\Lambda$, in the sense that there is an obvious natural collapse $\Upsilon \rightarrow \Lambda$. We often write $v(P)$ for the vertex $v \in \Lambda$ such that $F_{v}$ contains $P$. We call $X_{\mathcal{P}}=X-\mathcal{H}_{\mathcal{Z}}$ the cut decomposition of $X$.

For every index $j \in\{1,2, \cdots, n+2\}$, we denote the union of all the bind-type hyperplane of index $j$ as:

$$
\mathcal{H}^{j} \subset X
$$

Lemma 4.12. For every index $j \in\{1,2, \cdots, n+2\}$, there are only finitely many index- $j$ bind-type hyperplanes in $X$. Moreover, index- $j$ bind-type hyperplanes are all two-sided, canonically oriented, so that $\mathcal{H}^{j}$ is dual to $\xi^{j}$ in $H^{1}(X) \cong H^{1}\left(M_{\sigma}\right)$.

Proof. Because the bind-curves $\mathcal{W}$ are canonically oriented (Definition 4.3), and because edges in $\mathbf{R}^{n+2}$ are naturally directed by the basis vectors, it is clear that $\mathcal{H}^{j}$ is two-sided and canonically oriented.

To see there are only finitely many bind-type hyperplanes in $X$, observe that every cut-type hyperplane, as an $(n+2)$-dimensional cube complex by itself, has only finitely many sub-hyperplanes. In fact, let $H_{z} \subset X$ be the cut-type hyperplane corresponding to the cut-curve $z \subset \mathcal{Z}$. With the notations in the proof of Lemma 4.11, the element in $\pi_{1}\left(T_{z}\right)$ represented by the cut-curve $z$ acts on $\widehat{z} \times \mathbf{R}_{i(v)}^{n+1}$ as a translation by $\vec{\xi}(z)=\left(\xi^{1}([z]), \cdots, \xi^{n+2}([z])\right)=\vec{\Delta}(\bar{\xi}([z]))$, which is nontrivial on every component. Thus for every $j \neq i(v)$ in $\{1, \cdots, n+2\}$, there are only finitely many distinct sub-hyperplanes in $H_{z}$, which are projected by a parallel copy of $j$-th coordinate sub-hyperplane $\mathbf{R}_{(i(v), j)}^{n}$, namely, the intersection of $\mathbf{R}_{(i(v))}^{n+1}$ and $\mathbf{R}_{(j)}^{n+1}$. This means $H_{z}$ has only finitely many sub-hyperplanes. This finiteness implies the finiteness of bind-type hyperplanes in $X$, because every bind-type hyperplane must intersect at least one $H_{z}$ in a (nonempty) sub-hyperplane by our construction.

Counting the algebraic intersection of $\mathcal{H}^{j}$ with any representative cycle of $H_{1}(X)$ induces a dual cohomology class in $H^{1}(X) \cong \operatorname{Hom}\left(H_{1}(X), \mathbf{Z}\right)$. To see that $\mathcal{H}^{j}$ is dual to $\xi^{j}$, observe that the restriction of $\xi^{j}$ to each $H^{1}\left(X_{v}\right)$ is dual to $\mathcal{H}^{j} \cap X_{v}$. 
This follows immediately from the construction of $X_{v}$. Furthermore, it is also clear from our canonical gluing that for any lift $h: \Lambda \rightarrow X$ of the configuration graph, $\xi^{j}$ evaluates on the image of $H_{1}(\Lambda)$ precisely by counting the algebraic intersection number of any representative cycle with $\mathcal{H}^{j}$. Note that the image of $H_{1}(\Lambda)$ and all the $H_{1}\left(X_{v}\right)$ 's generate $H_{1}(X)$. Therefore, $\mathcal{H}^{j}$ is dual to $\xi^{j} \in H^{1}(X)$.

While they are complicated in general, bind-type hyperplanes are locally easy to understand. Note that for any bind-type hyperplane $H$ in $X$, and for any pairof-pants $P \subset \mathcal{P}$, the components of $H \cap X_{P}$ are all hyperplanes in $X_{P}$, with the bind-type index known as that of $H$. The vertical index $i(P)$ with respect to $P$ is known as the vertical index $i(v)$ with respect to the unique $v \in \operatorname{Ver}(\Lambda)$ such that $P \subset F_{v}$; and the other indices are the horizontal indices with respect to $P$. A bind-type hyperplane in $X_{P}$ of index $j$ is said to be vertical if its index $j$ equals $i(P)$; it is said to be horizontal, if $j$ differs from $i(P)$.

Lemma 4.13. For every pair-of-pants $P \subset \mathcal{P}$, the vertical hyperplanes in $X_{P}$ are in natural bijection to the bind-arcs in $P$, namely, the components of $P \cap \mathcal{W}$. Moreover, each vertical hyperplane in $X_{P}$ is homeorphic to $A^{2} \times \mathbf{R}^{n}$, with the two boundary components lying on two distinct components of $\partial X_{P}$. Here $A^{2}$ denotes the compact annulus.

Proof. Note that the universal covering $\widehat{X}_{P}$ is $\widehat{P} \times \mathbf{R}_{(i(P))}^{n+1}$, where $\widehat{P}$ is the universal covering of $P$. For every vertical hyperplane $Q \subset X_{P}$, every component $\hat{Q} \subset \widehat{X}_{P}$ of its preimage is the product $\hat{w} \times \mathbf{R}_{(i(P))}^{n+1}$, where $\hat{w} \subset \widehat{P}$ is a preimage-component of a unique bind-arc $w \subset P \cap \mathcal{W}$. The stabilizer of $\hat{Q}$ in $\pi_{1}\left(J_{P}\right)$ is $\pi_{1}\left(f_{v(P)}\right)$. This implies that $Q$ is homeomorphic to $A^{2} \times \mathbf{R}^{n}$. Moreover, different choices of the preimage-component $\hat{Q}$ projects to the same $Q \subset X_{v}$, so $Q \mapsto w$ is well-defined, sending every vertical hyperplane in $X_{P}$ to a bind-arc $w$ on $P$. It is straightforward to check that this defines a bijection between these two kinds of objects.

For every pair-of-pants $P \subset \mathcal{P}$, and for every horizontal index $j$, we denote the restriction of $\xi^{j}$ to $J_{P}$ as $\xi_{P}^{j} \in H^{1}\left(J_{P}\right)$, and the restriction of $\xi^{j}$ to $T_{z}$ as $\xi_{z}^{j} \in H^{1}\left(T_{z}\right)$. Remember also from Subsection 4.1 that $\bar{\xi} \in H^{1}(F)$ is the cohomology class dual to the bind-curves $\mathcal{W}$. We denote the restriction of $\bar{\xi}$ to $P$ as $\bar{\xi}_{P} \in H^{1}(P)$. For any nonzero integer $d, \bar{\xi}_{P}$ modulo $d$ lives in $H^{1}\left(P ; \mathbf{Z}_{d}\right)$, and it induces a dual cyclic covering of $P$ corresponding to the kernel of $\pi_{1}(P) \rightarrow H_{1}(P) \rightarrow \mathbf{Z}_{d}$, whose degree is the greatest common divisor of $d$ and the divisibility $\operatorname{div}\left(\bar{\xi}_{P}\right)$ of $\bar{\xi}_{P}$. Recall that the divisibility $\operatorname{div}(\xi)$ of a nontrivial element $\xi$ in a free abelian group $V$ is the largest positive integer by which $\xi$ is divisible in $V$.

Lemma 4.14. For every pair-of-pants $P \subset \mathcal{P}$, and for every horizontal index $j$, there are exactly $\operatorname{div}\left(\xi_{P}^{j}\right)$ index- $j$ horizonal hyperplanes in $X_{P}$. Moreover, these hyperplanes are all parallel, each homeomorphic to $\tilde{P}^{j} \times \mathbf{R}^{n}$, where $\tilde{P}^{j}$ is the finite cyclic covering of $P$ dual to $\bar{\xi}_{P}$ modulo $\xi^{j}\left(\left[f_{v(P)}\right]\right)$. In particular, for every cut-curve $z \subset \mathcal{Z}$ on $\partial P$, each horizontal hyperplane has exactly $\operatorname{div}\left(\xi_{z}^{j}\right) / \operatorname{div}\left(\xi_{P}^{j}\right)$ boundary components on the corresponding component of $\partial X_{P}$.

Proof. Let $Q \subset X_{P}$ be the horizontal hyperplane of index $j$. If $X_{v}$ is the piece containing $X_{P}$, then the construction of $X_{v}$ implies that $X_{P}$ is the quotient of its 
universal covering $\widehat{X}_{P} \cong \widehat{P} \times \mathbf{R}_{(i(P))}^{n+1}$ by the action $\pi_{1}\left(J_{P}\right)$ defined in the same manner, and $Q$ is projected from from $\widehat{P} \times \mathbf{R}_{(i(P), j)}^{n}$, where $\mathbf{R}_{(i(P), j)}^{n}$ for the intersection $\mathbf{R}_{(i(P))}^{n+1} \cap \mathbf{R}_{(j)}^{n+1}$. The stabilizer:

$$
G_{P}^{j}=\operatorname{Stab}_{\pi_{1}\left(J_{P}\right)}\left(\widehat{P} \times \mathbf{R}_{(i(P), j)}^{n}\right),
$$

consists of elements $g \in \pi_{1}(P)$ such that $\xi^{j}([g])=0$. Note that the canonical Seifert-fibration induces the commutative diagram of groups, where the rows are short exact sequences:

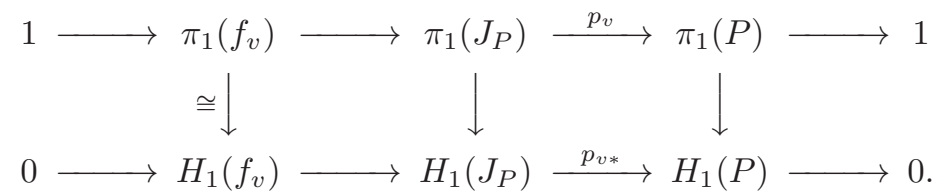

Regarding $\xi_{P}^{j}$ naturally as a linear functional in $\operatorname{Hom}\left(H_{1}\left(J_{P}\right), \mathbf{Z}\right)$, the stabilizer $G_{P}^{j}$ is precisely the preimage in $\pi_{1}\left(J_{P}\right)$ of $\operatorname{Ker}\left(\xi_{P}^{j}\right)$ with respect to the abelianization $\pi_{1}\left(J_{P}\right) \rightarrow H_{1}\left(J_{P}\right)$. Because $\xi^{j}\left(\left[f_{v}\right]\right)$ does not vanish, (Lemma 4.7), $G_{P}^{j} \cap \pi_{1}\left(f_{v}\right)$ is trivial, so $G_{P}^{j}$ embeds into $\pi_{1}(P)$ under $p_{v}$. This means $G_{v}^{j}$ acts properly on the $\widehat{P}$ factor of $\widehat{P} \times \mathbf{R}_{(i(P), j)}^{n}$, so $Q$ is homeomorphic to $\tilde{P}^{j} \times \mathbf{R}^{n}$, where $\tilde{P}^{j}$ is the normal covering of $P$ corresponding to the subgroup $p_{v}\left(G_{P}^{j}\right)$ of $\pi_{1}(P)$. Moreover,

$$
\begin{aligned}
\pi_{1}(P) / p_{v}\left(G_{P}^{j}\right) & \cong H_{1}(P) / p_{v *}\left(\operatorname{Ker}\left(\xi_{P}^{j}\right)\right) \\
& \cong H_{1}\left(J_{P}\right) /\left(H_{1}\left(f_{v}\right) \oplus \operatorname{Ker}\left(\xi_{P}^{j}\right)\right) \\
& \cong \operatorname{Im}\left(\xi_{P}^{j}\right) /\left(\xi_{P}^{j}\left(\left[f_{v}\right]\right) \mathbf{Z}\right) \\
& \cong \operatorname{Im}\left(\bar{\xi}_{P}\right) /\left(\xi_{P}^{j}\left(\left[f_{v}\right]\right) \mathbf{Z}\right) .
\end{aligned}
$$

From this we see that $\tilde{P}^{j}$ is a finite cyclic covering of $P$ dual to $\bar{\xi}_{P}$ modulo $\xi_{P}^{j}\left(\left[f_{v}\right]\right)$.

To see there are exactly $\operatorname{div}\left(\xi_{P}^{j}\right)$ copies of $Q$ in $X_{P}$, note that the parallel copies of $\widehat{P} \times \mathbf{R}_{(i(P), j)}^{n}$ are parametrized as $\mathbf{Z}$ by the $j$-th coordinate of $\mathbf{R}^{n+2}$. The action of $\pi_{1}\left(J_{P}\right)$ on the spaces of these copies is exactly the translation group generated by a translation of $\operatorname{distance} \operatorname{div}\left(\xi_{P}^{j}\right)$.

Finally, the number of boundary components of $Q$ on the boundary component $H_{z} \subset \partial X_{P}$ corresponding to $z \subset \partial P$ is the index of the image $\pi_{1}(z)$ in $\pi_{1}(P) / p_{v}\left(G_{P}^{j}\right)$. In other words, it is the index of the subgroup of $\operatorname{Im}\left(\xi_{P}^{j}\right)$ generated by $\xi_{z}^{j}([z])$ and $\xi_{z}^{j}\left(\left[f_{v}\right]\right)$. Note $\operatorname{Im}\left(\xi_{P}^{j}\right)$ is identified as the $\operatorname{subgroup} \operatorname{div}\left(\xi_{P}^{j}\right) \mathbf{Z}$ of Z. Since $[z]$ and $\left[f_{v}\right]$ generates $H_{1}\left(T_{z}\right), \operatorname{div}\left(\xi_{z}^{j}\right)$ is just the greatest common divisor of $\xi_{z}^{j}([z])$ and $\xi_{z}^{j}\left(\left[f_{v}\right]\right)$. Hence the index equals $\operatorname{div}\left(\xi_{z}^{j}\right) / \operatorname{div}\left(\xi_{P}^{j}\right)$.

In order to capture the global geometry of bind-type hyperplanes in $X$, it is useful to consider its decomposition induced from the cut decomposition of $X$. Specifically, let $H \subset X$ be a bind-type hyperplane. There is a naturally associated graph $\Upsilon_{H}$, whose vertices correspond to the components of $H \cap X_{\mathcal{P}}$, and whose edges correspond to the components of $H \cap \mathcal{H}_{\mathcal{Z}}$. Hence there is a naturally associated 
map $\iota_{H}: \Upsilon_{H} \rightarrow \Upsilon$ between graphs, satisfying the following commutative diagram:

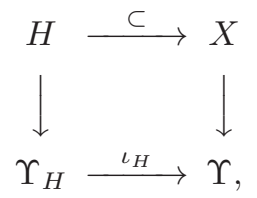

where the vertical maps are natural collapses. Note also that $\Upsilon_{H}$ is a finite graph by the proof of Lemma 4.12 .

Lemma 4.15. For a bind-type hyperpane $H$ of $X$, the associated map $\iota_{H}: \Upsilon_{H} \rightarrow \Upsilon$ is an immersion (i.e. a local isometry) if and only if no component of any $H \cap X_{P}$ has two boundary components lying on the same component of $\partial X_{P}$; and $\iota_{H}$ is an embedding if and only if $H \cap X_{P}$ is connected (possibly empty) for every $X_{P}$.

Proof. This follows immediately from the definition.

We discuss which pathologies may occur to hyperplanes in $X$. In other words, we study the position relationship of hyperplanes near vertices. There are two types of vertices in $X$, called the octagon type and the band type. An octagon-type vertex comes from the center of the octagon in a pair-of-pants, while a band-type vertex comes from the center of a band in a pair-of-pants, (cf. Figure 2).

Lemma 4.16. The following statements are true.

(1) Every hyperplane in $X$ is two-sided with no self-intersection;

(2) Any two distinct hyperplanes of the same type do not intersect, and hence do not inter-osculate;

(3) Any two bind-type hyperplanes of distinct indices do not osculate, and hence do not inter-osculate.

Proof. The first statement has been proved in Lemma 4.11, Lemma 4.12. The second statement is true by the definition of types. The third statement is true by the definition of osculation. In fact, it is straightforward to check at each octagontype vertex and at each band-type vertex, that two edges dual to two bind-type hyperplanes of distinct indices, respectively, always share a common square.

There remains to be three kinds of possible pathologies:

- Self-osculation of cut-type hyperplanes;

- Self-osculation of bind-type hyperplanes;

- Inter-osculation between cut-type hyperplanes and bind-type hyperplanes.

To be more illustrative, let us name the edges in Figure 2 adjacent to the octagon center by the compass directions, such as the east, the northwest, etc. Selfosculation of cut-types occurs at every octagon-type vertex, as the north and the south are dual to the same cut-type hyperplane. Self-osculation of bind-types occurs at an octagon-type vertex, when the northwest (resp. southwest) and the southeast (resp. northeast) are dual to the same bind-type hyperplane. Inter-osculation between mixed types may also occur. For instance, if the northwest and the northeast are dual to the same bind-type hyperplane, this hyperplane will inter-osculate both the cut-type hyperplanes dual to the east and the west. 
4.4. Virtual specialness of $X$. In this subsection, we show the cube complex $X$ constructed in Subsection 4.2 is virtually special, namely, such that a finite covering of $X$ is a special cube complex (Definition 2.3).

Note that for any covering $\kappa: \tilde{X} \rightarrow X$, it makes sense to speak of the types of hyperplanes and vertices in $\tilde{X}$, according to the type of the underlying image. There will also be an induced cut decomposition of $\tilde{X}$, over a graph $\tilde{\Upsilon}$ whose vertices correspond to the components of the $\kappa^{-1}\left(X_{\mathcal{P}}\right)$, and whose edges correspond to the components of $\kappa^{-1}\left(\mathcal{H}_{\mathcal{Z}}\right)$. Moreover, for any bind-type hyperplane $\tilde{H}$ of $\tilde{X}$, there is also an associate decomposition graph $\tilde{\Upsilon}_{\tilde{H}}$, which is a finite graph, and there is an associated map $\iota_{\tilde{H}}: \tilde{\Upsilon}_{\tilde{H}} \rightarrow \tilde{\Upsilon}$, for which the conclusion of Lemma 4.15 still holds.

Our strategy is as follows. For every index $j \in\{1,2, \cdots, n+2\}$, pass to a finite cyclic covering $\tilde{X}^{j}$ of $X$, so that there is no self-osculation of cut-type hyperplanes in an $X_{P}$ with the vertical index $i(P)$ equal to $j$, and that every bind-type hyperplane of index $j$ in $\tilde{X}^{j}$ induces an immersion between the decomposition graphs. Then we pass to a further finite regular covering $\ddot{X}^{j}$ induced by a finite regular covering of the decomposition graph, so that every bind-type hyperplane of index $j$ in $\ddot{X}^{j}$ induces an embedding between the decomposition graphs. This will eliminate selfosculations of index- $j$ bind-types as well as inter-osculations between cut-types and index- $j$ bind-types. It follows that a common finite regular covering $\ddot{X}$ of all these $\ddot{X}^{j}$ 's will be special.

Step 1. The finite cyclic covering $\tilde{X}^{j}$. Let $j \in\{1,2, \cdots, n+2\}$ be an index. Note that for every cut-curve $z \subset \mathcal{Z}$, the restricted cohomology class $\xi_{z}^{j} \in H^{1}\left(T_{z}\right)$ is nontrivial as it evaluates nontrivially on $[z] \in H_{1}\left(T_{z}\right)$, (Lemmas 4.5, 4.7). Let the positive integer $l^{j}$ be the least common multiple of all the divisibilities $\operatorname{div}\left(\xi_{z}^{j}\right)$ as $z$ runs over the all components of the cut-curves $\mathcal{Z}$. Let:

$$
\tilde{\kappa}^{j}: \tilde{X}^{j} \rightarrow X,
$$

be the finite cyclic covering corresponding to the kernel of the the composed homomorphism:

$$
\pi_{1}\left(M_{\sigma}\right) \stackrel{[\cdot]}{\longrightarrow} H_{1}\left(M_{\sigma}\right) \stackrel{\xi^{j}}{\longrightarrow} \mathbf{Z} \stackrel{\bmod l^{j}}{\longrightarrow} \mathbf{Z}_{l^{j}} .
$$

It is clear that the covering degree is $l^{j} / \operatorname{div}\left(\xi^{j}\right)$. We denote the induced decomposition graph of $\tilde{X}^{j}$ as $\tilde{\Upsilon}^{j}$.

Lemma 4.17. For any pair-of-pants $P \subset \mathcal{P}$ with the vertical index $i(P)$ equal to $j$, and for any vertex $\tilde{\nu} \in \tilde{X}^{j}$ with $\tilde{\kappa}^{j}(\tilde{\nu}) \in X_{P}$, no cut-type hyperplane in $\tilde{X}^{j}$ self-osculates at $\tilde{\nu}$.

Proof. By Lemma 4.11, cut-type hyperplanes do not self-osculate at any band-type vertex in $X$. In fact, there are two cut-type edges adjacent to each band-type vertex, but they are dual to different hyperplanes. Thus cut-type hyperplanes do not self-osculate at any band-type vertex in $\tilde{X}^{j}$ either.

Suppose there were an octagon-type vertex $\tilde{\nu} \in \tilde{X}^{j}$, such that $\tilde{\kappa}^{j}(\tilde{\nu}) \in X_{P}$ for some pair-of-pants $P \subset \mathcal{P}$ with the vertical index $i(P)$ equal to $j$, and that there are two cut-type edges adjacent to it dual to the same cut-type hyperplane $\tilde{H} \subset \tilde{X}^{j}$. Pick a path on $\tilde{H}$ joining the two end-points on $\tilde{H}$ of the two edges. Project the loop $\tilde{c}$ formed by the path and the two edges to $X$ via $\tilde{\kappa}^{j}$, then $c=\tilde{\kappa}^{j}(\tilde{c})$ represents an element in $H_{1}(X)$. Let $z \subset \mathcal{Z}$ be the cut-curve whose corresponding hyperplane $H_{z} \subset X$ equals $\tilde{\kappa}^{j}(\tilde{H})$, (Lemma 4.11). Then by counting the algebraic intersection 
number of $c$ and $\mathcal{H}^{j}, \xi^{j}([c])$ modulo $\xi^{j}([z])$ is nontrivial. Note that the absolute value of $\xi^{j}([z])$ is the divisibility of $\xi_{z}^{j}$, since $H_{1}\left(T_{z}\right)$ is generated by $[z]$ and $\left[f_{v}\right]$ while $\xi^{j}\left(\left[f_{v}\right]\right)=0$, (Lemma 4.7). Thus $\xi^{j}([c])$ modulo $l^{j}$ is nontrivial either. Such a loop $\tilde{c}$ cannot lie in the covering space $\tilde{X}^{j}$, so we reach a contradiction.

Lemma 4.18. For every index-j bind-type hyperplane $\tilde{H} \subset \tilde{X}^{j}$, the associated map between the decomposition graphs:

$$
\iota_{\tilde{H}}: \tilde{\Upsilon}_{\tilde{H}}^{j} \rightarrow \tilde{\Upsilon}^{j},
$$

is an immersion.

Proof. By Lemma 4.15, we must show that for any component $\tilde{X}_{P} \subset \tilde{X}^{j}$ of the preimage $\left(\tilde{\kappa}^{j}\right)^{-1}\left(X_{P}\right)$, no two boundary components of $\tilde{Q}=\tilde{H} \cap \tilde{X}_{P}$ lie in the same component of $\partial \tilde{X}_{P}$.

If $\tilde{Q}$ is vertical in $\tilde{X}_{P}$, this is automatically true by Lemma 4.13 .

If $\tilde{Q}$ is horizontal in $\tilde{X}_{P}$, suppose there were some component $\tilde{H} \subset \partial \tilde{X}_{P}$ containing two distinct boundary components $\tilde{U}, \tilde{U}^{\prime} \subset \tilde{Q}$. Let $z \subset \mathcal{Z}$ be the cut-curve so that $H_{z} \subset X$ equals $\tilde{\kappa}^{j}(\tilde{H})$. Because $\tilde{\kappa}^{j}$ is the cyclic covering induced by $\xi^{j}$ modulo $l^{j}$, and because the union $\mathcal{H}^{j} \subset X$ of index- $j$ bind-type hyperplanes is dual to $\xi^{j}$ by our construction, (Lemma 4.12), $\tilde{X}^{j}$ can be obtained by cyclically gluing $l^{j} / \operatorname{div}\left(\xi^{j}\right)$ copies of $X-\mathcal{H}^{j}$. In particular, $\tilde{\kappa}^{j}$ embeds $\tilde{Q}^{j}$ into $X_{P}$, so the projected images of $\tilde{U}$ and $\tilde{U}^{\prime}$ in $X_{v}$ are different as well. Pick a path in $\tilde{Q}$ with the two end-points $\tilde{\nu}, \tilde{\nu}^{\prime}$ on $U, U^{\prime}$, respectively, and pick another path in $\tilde{H}$ joining $\tilde{\nu}, \tilde{\nu}^{\prime}$. Project the loop $\tilde{c}$ formed by these two paths into $X_{P}$, so that $c=\tilde{\kappa}^{j}(\tilde{c})$ represents an element in $H_{1}\left(X_{v}\right)$. As $\tilde{U}, \tilde{U}^{\prime}$ have distinct projected images, counting the algebraic intersection number of $c$ with $\mathcal{H}^{j}$ yields that $\xi^{j}([c]) \operatorname{modulo} \operatorname{div}\left(\xi_{z}^{j}\right) / \operatorname{div}\left(\xi_{P}^{j}\right)$ is nontrivial, (Lemma 4.14). Thus $\xi^{j}([c]) \bmod l^{j}$ is nontrivial either, which leads to a contradiction as the loop $\tilde{c}$ cannot lie in the covering space $\tilde{X}^{j}$.

Step 2. The graph-induced covering $\ddot{X}^{j}$. We find some finite regular covering $\ddot{\Upsilon}^{j} \rightarrow \tilde{\Upsilon}^{j}$, so that for every bind-type hyperplane of index $j$, all the elevations of $\iota_{\tilde{H}}$ into $\ddot{\Upsilon}$ are embeddings. Here by an elevation of $\iota_{\tilde{H}}$ we mean a map $\iota_{\ddot{H}}: \ddot{\Upsilon}_{\ddot{H}}^{j} \rightarrow \ddot{\Upsilon}^{j}$ from a connected covering $\ddot{\Upsilon}_{\ddot{H}}^{j}$ of $\tilde{\Upsilon}_{\tilde{H}}^{j}$ into $\ddot{\Upsilon}^{j}$, minimal subject to the following commutative diagram of maps:

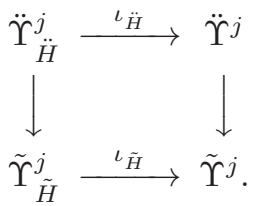

Writing $\ddot{X} \rightarrow \tilde{X}$ for the naturally induced finite regular covering of $\tilde{X}$, it is clear that every elevation is exactly the induced map $\iota_{\ddot{H}}$ for some component $\ddot{H}$ of the preimage of $\tilde{H}$.

Lemma 4.19. There exists a finite regular covering $\ddot{\Upsilon}^{j} \rightarrow \tilde{\Upsilon}^{j}$, so that for every bind-type hyperplane of index $j$, every elevation of $\iota_{\tilde{H}}$ into $\ddot{\Upsilon}^{j}$ is an embedding.

Proof. This follows from the fact that finitely generated free groups are LERF, ([Sc, Theorem 2.2]). Recall from [Sc] that a group $G$ is said to be locally extended residually finite (or $L E R F$ ), if for every finitely generated subgroup $H$ of $G$, and 
for every $g \in G$ not in $H$, there is a finite-index subgroup $\tilde{G}$ of $G$ containing $H$, which does not contain $g$. If $S$ is a connected CW complex with $\pi_{1}(S) \cong G$, then $G$ is LERF if and only if for every finitely generated covering $\kappa: \tilde{S} \rightarrow S$ and every compact subset $K \subset \tilde{S}, \kappa$ factors through a finite covering of $S$ in which $K$ embeds, (cf. [Sc, Lemma 1.4]).

Suppose $\tilde{H} \subset \tilde{X}^{j}$ is a bind-type hyperplane of index $j$, by Lemma $4.18, \iota_{\tilde{H}}$ : $\tilde{\Upsilon}_{\tilde{H}}^{j} \rightarrow \tilde{\Upsilon}^{j}$ is an immersion, so it is $\pi_{1}$-injective. Let $\operatorname{Cov}\left(\iota_{\tilde{H}}\right) \rightarrow \tilde{\Upsilon}^{j}$ be the covering corresponding to image of $\left(\iota_{\tilde{H}}\right)_{\sharp}: \pi_{1}\left(\tilde{\Upsilon}_{\tilde{H}}^{j}\right) \hookrightarrow \pi_{1}\left(\tilde{\Upsilon}^{j}\right)$, (after picking a base-point). Then $\iota_{\tilde{H}}$ elevates to an embedding into $\operatorname{Cov}\left(\iota_{\tilde{H}}\right)$. Because $\pi_{1}\left(\tilde{\Upsilon}^{j}\right)$ is a finitely generated free group, it is $\operatorname{LERF}$, so $\operatorname{Cov}\left(\iota_{\tilde{H}}\right) \rightarrow \tilde{\Upsilon}^{j}$ factors through a finite covering $\Psi^{\tilde{H}}$, in which an elevated image $\tilde{\Upsilon}_{\tilde{H}} \hookrightarrow \Psi^{\tilde{H}}$ embeds. In other words, there is an elevation of $\iota_{\tilde{H}}$ into $\Psi^{\tilde{H}}$ which is an embedding. Pass to the characteristic covering $\ddot{\Upsilon}^{j, \tilde{H}} \rightarrow \tilde{\Upsilon}^{j}$, corresponding to the intersection of all the conjugates of $\pi_{1}\left(\Psi^{\tilde{H}}\right)$ in $\pi_{1}\left(\tilde{\Upsilon}^{j}\right)$, then $\ddot{\Upsilon}^{j, \tilde{H}}$ is a finite regular covering into which every elevation of $\iota_{\tilde{H}}$ is an embedding.

By Lemma 4.12, there are only finitely many bind-type hyperplanes of index $j$ in $X$, so there are only finitely many index- $j$ bind-types in $\tilde{X}^{j}$ as well. We take a common finite regular covering $\ddot{\Upsilon}^{j}$ of all these $\ddot{\Upsilon}^{j, \tilde{H}}$. It is the covering as desired.

Let:

$$
\ddot{\kappa}^{j}: \ddot{X}^{j} \rightarrow \tilde{X}^{j},
$$

be the finite regular covering of $\tilde{X}^{j}$ naturally induced from the finite regular covering $\ddot{\Upsilon}^{j} \rightarrow \tilde{\Upsilon}^{j}$ as in Lemma 4.18 .

Lemma 4.20. No bind-type hyperplane of index $j$ self-osculates in $\ddot{X}^{j}$.

Proof. By Lemmas 4.13, 4.14, no bind-type hyperplane in any $X_{P}$ self-osculates. Indeed, bind-type edges adjacent at any vertex are dual to distinct hyperplanes in $X_{P}$. Passing to the covering space, this also holds for any component of $\left(\ddot{\kappa}^{j}\right)^{-1}\left(X_{P}\right)$. Now let $\ddot{H} \subset \ddot{X}^{j}$ be any hyperplane. Suppose $\ddot{X}_{P}$ is any component of $\left(\ddot{\kappa}^{j}\right)^{-1}\left(X_{P}\right)$, for any $P \subset \mathcal{P}$. By Lemmas 4.15, 4.19, $\ddot{H}$ intersects $\ddot{X}_{P}$ in a single hyperplane, if not in the empty set. Thus at any vertex in $\ddot{X}_{P}$, at most one edge is dual to $\ddot{H}$ in $\ddot{X}^{j}$. This means $\ddot{H}$ does not self-osculate in $\ddot{X}^{j}$, which completes the proof.

Lemma 4.21. No bind-type hyperplane of index $j$ inter-osculates any cut-type hyperplane in $\ddot{X}^{j}$.

Proof. By Lemma 4.11, every cut-type hyperplane of $X$ is contained in exactly two components of $X_{\mathcal{P}}$. This also holds for cut-type hyperplanes in $\ddot{X}^{j}$. If a cut-type hyperplane $\ddot{S} \subset \ddot{X}$ intersect an index- $j$ bind-type hyperplane $\ddot{H} \subset \ddot{X}^{j}$. Suppose $\ddot{X}_{P}$ is a component of some $(\ddot{\kappa})^{-1}\left(X_{P}\right)$, which is adjacent to $\ddot{S}$. It follows from Lemmas 4.15, 4.19 that the intersection $\ddot{H} \cap \ddot{X}_{P}$ is a single hyperplane in $\ddot{X}_{P}$, and it does not osculate $\ddot{S}$, (Lemmas $4.13,4.14$ ). This implies $\ddot{H}$ does not osculate $\ddot{S}$, which completes the proof.

Step 3. The common covering $\ddot{X}$. We take the covering:

$$
\ddot{\kappa}: \ddot{X} \rightarrow X,
$$


to be the common covering of all the $\ddot{X}^{j}$, as $j$ runs over $\{1,2, \cdots, n+2\}$. In other words, $\ddot{X}$ is the finite regular covering of $X$ corresponding to the intersection of all the subgroups $\pi_{1}\left(\ddot{X}^{j}\right)$ of $\pi_{1}(X)$.

Lemma 4.22. The cube complex $\ddot{X}$ is special.

Proof. By Lemma 4.16 (1), every hyperplane in $\ddot{X}$ is two-sided with no self-intersection. By Lemmas 4.17, 4.20 and the fact that $\ddot{X}$ is the common covering of all the $\ddot{X}^{j}$ 's, no hyperplane in $\ddot{X}$ self-osculates. By Lemmas 4.16 (2), (3), 4.21, there is no interosculation between hyperplanes in $\ddot{X}$ either. We conclude that the cube complex $\ddot{X}$ is special.

To sum up, we have shown that for a multitwist $(F, \sigma)$ with a bipartite configuration graph, if the current equations associated to $(F, \sigma)$ have a nondegenerate symmetric solution, then we can find a cut-bind system $(\mathcal{Z}, \mathcal{W})$, (Subsection 4.1), and construct a canonical cube complex $X$, (Subsection 4.2). Moreover, $X$ is homeomorphic to $M_{\sigma} \times \mathbf{R}^{n}$ for some $0 \leq n \leq|\operatorname{Ver} \Lambda|-2$, (Lemma 4.10), and $X$ is virtually special, (Lemma 4.22).

This completes the proof of Proposition 4.1.

\section{Proof of the Main theorem}

In this section, we summarize the proof of Theorem 1.1.

Lemma 5.1. Theorem 1.1 holds for aspherical compact Seifert fibered spaces.

Proof. A compact Seifert space is aspherical if and only if its base orbifold is compact and aspherical. It is virtually fibered if and only if either it has nonempty boundary, or that it is closed with vanishing Euler class of the fiber. Note that this is exactly the case when the Seifert fibered space is nonpositively curved, cf. [KL, GW]. The other three statements are straightforward to check.

Lemma 5.2. None of the statements in Theorem 1.1 is true if $M$ is virtually the mapping torus of an Anosov torus automorphism. Hence they are equivalent in this case.

Proof. Such an $M$ is Sol-geometric, so it is not nonpositively curved, ([GW], [Ya]). It is clearly not virtually RFRS either. In fact, for any sequence of finite index subgroups $\pi_{1}(M)=G_{0} \triangleright G_{1} \triangleright \cdots$ with each $G_{i} \rightarrow G_{i} / G_{i+1}$ factoring through a free abelian group, the intersection of all the $G_{i}$ 's always contains a finite-index subgroup the commutator subgroup of $\pi_{1}(M)$. Note that the second and the third statement implies the fourth, (cf. Subsections 2.4, 2.5). Thus none of the statements in Theorem 1.1 holds for such an $M$.

Lemma 5.3. If the conclusion of Theorem 1.1 holds for the mapping torus $M_{\sigma}$ of every multitwist $(F, \sigma)$ with a bipartite configuration graph, it holds for any nontrivial compact graph manifold as well.

Proof. The bounded case reduces to the closed case. In fact, if $M$ is a compact graph manifold with nonempty boundary, it follows from [Le] that $M$ supports a nonpositively curved Riemannian metric with totally geodesic boundary. Then the double $W$ of $M$ along the boundary is closed and nonpositively curved. Assuming that Theorem 1.1 holds in the closed case, then $\pi_{1}(W)$ is virtually specially cubulated, and is virtually a subgroup of a right-angled Artin group, and is virtually 
RFRS. As $\pi_{1}(M)$ may be regarded as a subgroup of $\pi_{1}(W)$, the same holds for $\pi_{1}(M)$.

The closed case reduces to the fibered case. This is because all the statements of Theorem 1.1 are virtual properties, and each of them implies that the manifold is virtually fibered, (Theorems 2.1, 2.4, 2.6). Furthermore, Lemmas 3.3, 5.2 imply that it suffices to prove Theorem 1.1 for the mapping torus of multitwists with a bipartite configuration graphs.

After these reductions, we are ready to prove Theorem 1.1.

Proof of Theorem 1.1. We reduced to the case for nontrivial graph manifolds by Lemma 5.1. Moreover, by Lemma 5.3, it suffices to assume that $M$ is the mapping torus $M_{\sigma}$ of some multitwist $(F, \sigma)$ with a bipartite configuration graph.

To see $(1) \Rightarrow(2)$, note the nonpositive curving implies a nondegenerate symmetric solution of the BKN equations associated to $(F, \sigma)$, (Theorem 3.8). By Proposition 3.9, the current equations virtually have a nondegenerated symmetric solution as well. After passing to a finite covering, we can apply Proposition 4.1 to construct a special cube complex homotopy equivalent to $M_{\sigma}$.

To see $(2) \Rightarrow(3)$, we apply Theorem 2.4. Note that $\pi_{1}\left(M_{\sigma}\right)$ is finitely generated, so we may further require the right-angled Artin group $A(\Gamma)$ to be finitely generated, for instance, by taking subgroup $A\left(\Gamma^{\prime}\right)$ generated only by the generators of $A(\Gamma)$ that spell the words representing the image of the generators of $\left(M_{\sigma}\right)$.

To see $(3) \Rightarrow(4)$, observe that finitely generated Artin groups are all virtually RFRS, (cf. Subsection 2.5).

To see $(4) \Rightarrow(1)$, note the virtual RFRS-ness implies virtually a nondegenerate symmetric solution of the current equations, (Lemma 3.6), and hence a nondegenerate symmetric solution to the BKN equations, (Proposition 3.9). By Theorem $3.8, M_{\sigma}$ is nonpositively curved. This completes the proof.

\section{REFERENCES}

[Ag1] I. Agol, Criteria for virtual fibering, J. Topol. 1 (2008), 269-284.

[Ag2] I. Agol, The virtual Haken conjecture, with an appendix by I. Agol, D. Groves, J. Manning, preprint, 2012, arXiv:1204.2810.

[BS] S. V. Buyalo, P. V. Svetlov, Topological and geometric properties of graph manifolds, Algebra i Analiz 16 (2004), no. 2, 3-68.

[DJ] M. Davis, T. Januszkiewicz, Right-angled Artin groups are commensurable with right-angled Coxeter groups, J. Pure Appl. Algebra 153 (2000), no. 3, 229-235.

[Gr] M. Gromov, Hyperbolic groups, Essays in Group Theory, Math. Sci. Res. Inst. Publ. 8, Springer, NY, 1987, pp. 75-263.

[GW] D. Gromoll, J. A. Wolf, Some relations between the metric structure and the algebraic structure of the fundamental group in manifolds of nonpositive curvature, Bull. Amer. Math. Soc. 77 (1971), 545-552.

[HW] F. Haglund, D. T. Wise, Special cube complexes, Geom. Funct. Anal. 17 (2008), 1551-1620.

$[\mathrm{HsW}] \mathrm{T}$. Hsu, D. T. Wise, On linear and residual properties of graph products, Michigan Math. J. 46 (1999), no. 2, 251-259.

[Ja] W. Jaco, Lectures on Three-Manifold Topology, CBMS Regional Conference Series in Mathematics, 43. American Mathematical Society, Providence, RI, 1980.

[KL] M. Kapovich, B. Leeb, Actions of discrete groups on nonpositively curved spaces, Math. Ann. 306 (1996), no. 2, 341-352.

[Le] B. Leeb, 3-manifolds with(out) metrics of nonpositive curvature, Invent. Math. 122 (1995), 277-289. 
[LW] J. Luecke, Y.-Q. Wu, Relative Euler number and finite covers of graph manifolds, Geometric Topology (Athens, GA, 1993), AMS/IP Stud. Adv. Math., vol. 2.1, American Mathematical Society, Provi- dence, RI, 1997, pp. 80-103.

[Ne] W. D. Neumann, Immersed and virtually embedded $\pi_{1}$-injective surfaces in graph manifold, Algebr. Geom. Topol. 1 (2001), 411-426.

[Ni] B. Nica, Cubulating spaces with walls, Algebr. Geom. Topol. 4 (2004), 297-309.

[PW1] P. Przytycki, D. T. Wise, Graph manifolds with boundary are virtually special, in preparation.

[PW2] P. Przytycki, D. T. Wise, Mixed 3-manifolds are virtually special, preprint, 2012, arXiv: 1205.6742.

[RW] J. H. Rubinstein, S.-C. Wang, $\pi_{1}$-injective surfaces in graph-manifolds, Comment. Math. Helv. 73 (1998), 499-515.

[Sc] P. Scott, Subgroups of surface groups are almost geometric, J. London Math. Soc. (2) 17 (1978), no. 3, 555-565.

[Sv] P. V. Svetlov, Graph-manifolds of nonpositive curvature are virtually fibered over a circle, (Russian), Algebra i Analiz 14 (2002), no. 5, 188-201; English translation in St. Petersburg Math. J. 14 (2003), no. 5, 847-856.

[Th1] W. P. Thurston, Three dimensional manifolds, Kleinian groups and hyperbolic geometry, Bull. Amer. Math. Soc. (N.S.) 6 (1982), no. 3, 357-381.

[Th2] W. P. Thurston, On the geometry and dynamics of diffeomorphisms of surfaces, Bull. Amer. Math. Soc. 19 (1988), no. 2, 417-438.

[WY] S.-C. Wang, F.-C. Yu, Graph-manifolds with non-empty boundary are covered by a surface bundle over the circle, Math. Proc. Camb. Phil. Soc. 122 (1997), 447-455.

[Wi] D. T. Wise, Research announcement: the structure of groups with a quasiconvex hierarchy, Electron. Res. Announc. Math. Sci. 16 (2009), 44-55.

[Ya] S.-T. Yau, On the fundamental group of compact manifolds of non-positive curvature, Ann. of Math. (2) 93 (1971), no. 3, 579-585.

Mathematics 253-27, California Institute of Technology, Pasadena, CA 91125

E-mail address: yliumath@caltech.edu 